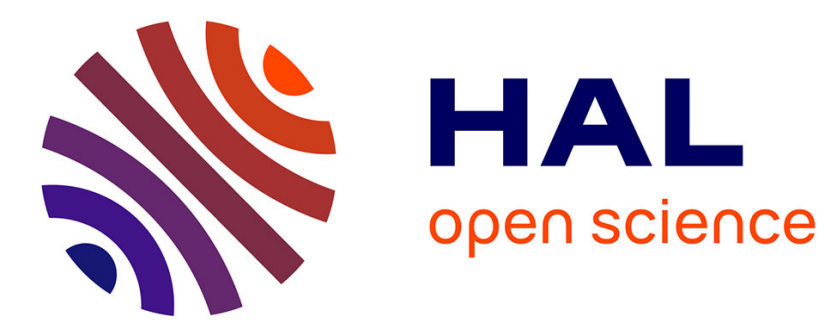

\title{
Les effets de l'activation des dépenses d'indemnisation chômage
}

\author{
Nadia Alibay, Arnaud Lefranc
}

\section{To cite this version:}

Nadia Alibay, Arnaud Lefranc. Les effets de l'activation des dépenses d'indemnisation chômage. Revue Française d'Economie, 2003, 18 (2), pp.55 - 110. 10.3406/rfeco.2003.1485 . halshs-01651809

\section{HAL Id: halshs-01651809 \\ https://shs.hal.science/halshs-01651809}

Submitted on 1 Apr 2020

HAL is a multi-disciplinary open access archive for the deposit and dissemination of scientific research documents, whether they are published or not. The documents may come from teaching and research institutions in France or abroad, or from public or private research centers.
L'archive ouverte pluridisciplinaire HAL, est destinée au dépôt et à la diffusion de documents scientifiques de niveau recherche, publiés ou non, émanant des établissements d'enseignement et de recherche français ou étrangers, des laboratoires publics ou privés. 


\section{Nadia ALIBAY \\ Arnaud LEFRANC}

\section{Les effets de}

\section{l'activation des dépenses}

d'indemnisation chômage

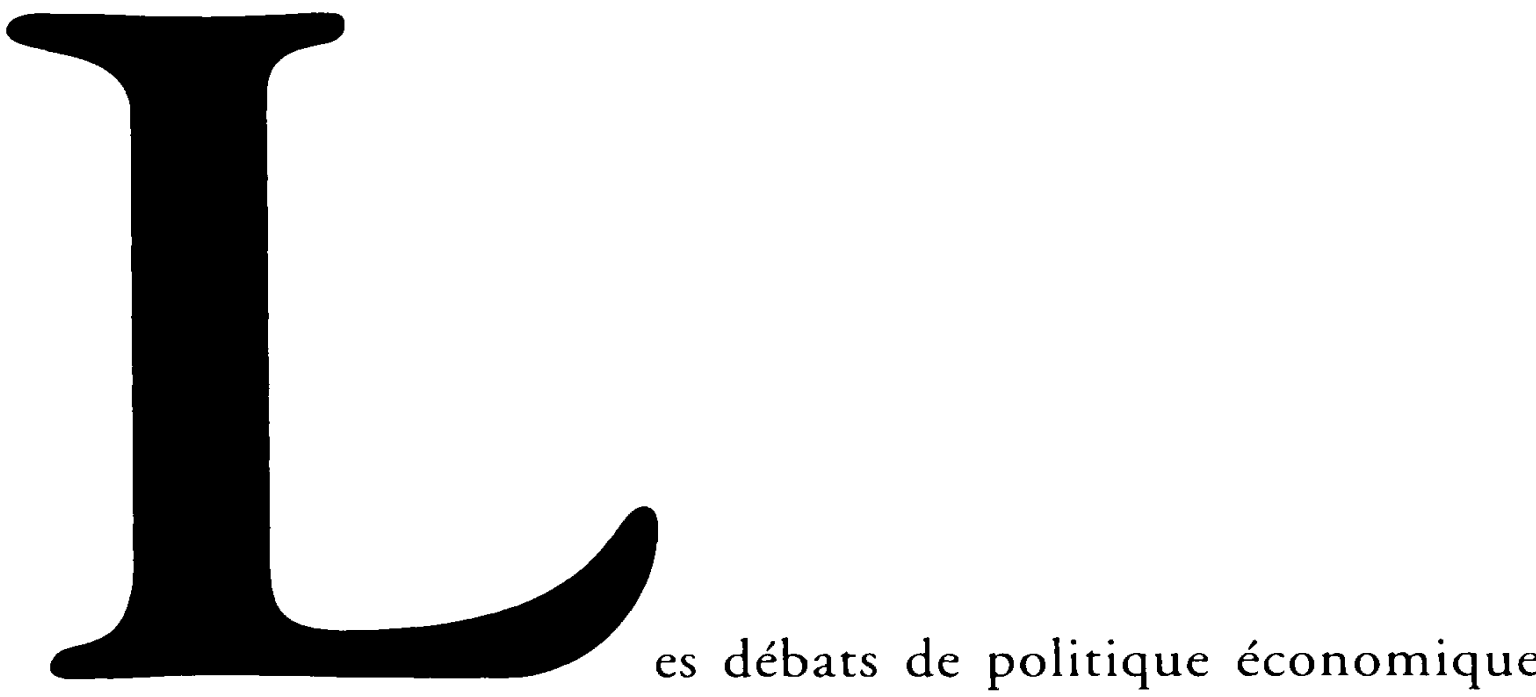
des années 1990 ont abondamment souligné les insuffisances des politiques dites passives du marché du travail, essentiellement réduites à l'indemnisation du chômage. Se limitant à traiter les conséquences individuelles du chômage, elles ne favoriseraient en aucun cas le retour à l'emploi des travailleurs les plus exposés au risque de chômage persistant, contrairement aux politiques actives de formation, d'aide à la recherche d'emploi et de subvention à l'emploi. En outre, elles pourraient avoir pour effet pervers de rendre peu attractif le retour à l'emploi 
des chômeurs indemnisés, contribuant ainsi à l'apparition de trappes à chômage.

Le développement des politiques actives du marché du travail se heurte cependant, dans la plupart des économies européennes, à deux contraintes majeures et difficilement conciliables : d'une part, la nécessité de maintenir dans des proportions raisonnables le budget de la politique de l'emploi ; d'autre part, le souhait de ne pas remettre en cause le principe d'assurance et les niveaux d'indemnisation des dispositifs en place.

Face à ces deux contraintes, l'idée d' " activation des dépenses passives " suggère qu'il est possible d'atteindre certains objectifs habituellement dévolus à la politique active, par une réforme adéquate des systèmes d'assurance chômage. Deux voies d'activation des dépenses passives ont, en pratique, été expérimentées. La première consiste à verser, en cas de sortie du chômage, une partie du reliquat de droits assurantiels, soit au salarié, soit à l'employeur. La seconde autorise un cumul partiel des indemnités chômage et des revenus salariaux d'emplois transitoires, de durée limitée et/ou à horaire de travail réduit. Dans les deux cas, ces réformes visent à limiter les effets désincitatifs de l'assurance-chômage. Elles rejoignent par ailleurs, dans leurs intentions, les politiques actives : politiques de subvention à l'emploi pour la première forme d'activation, politiques visant à renforcer l'employabilité des chômeurs par des mesures de formation en emploi pour la seconde.

Ces deux modes d'activation ont été intégrés à la politique de l'emploi française : le premier, dans le cadre Contrat de coopération de l'Unedic, le second, au sein du dispositif ARAC (activité réduite, allocation chômage) de l'Unedic. Si le nombre de bénéficiaires du Contrat de coopération, aujourd'hui supprimé, a toujours été très faible ${ }^{1}$, il n'en est pas de même pour le dispositif ARAC. Depuis sa mise en place en 1986, le nombre d'allocataires de l'assurance-chômage ayant parallèlement une activité rémunérée a fortement cru pour atteindre 340000 personnes au mois de décembre 2000 , soit $20 \%$ des chômeurs indemnisés. Plus largement, faisant écho à cette tendance, on assiste au sein de la population inscrite auprès de l'ANPE, au déve- 
loppement de situations intermédiaires au regard du marché du travail, combinant activité rémunérée et recherche d'emploi : ainsi, parmi les inscrits à l'ANPE, plus d'une personne sur quatre a déjà exercé une activité réduite pendant l'épisode en cours de recherche d'emploi.

Face au nombre important des bénéficiaires, il convient de s'interroger sur les effets de ce dispositif encore peu étudié et mal connu. L'a priori qui préside au développement de ces possibilités de cumul est qu'en augmentant les gains monétaires associés au retour à l'emploi, ce dispositif aura pour effet d'encourager les chômeurs à une reprise d'activité conduisant, à terme, à une insertion plus durable au marché du travail. On peut cependant opposer à ces effets, potentiellement favorables en termes d'insertion, la possibilité de phénomènes de précarisation. Dans la mesure où le dispositif existant encourage l'exercice d'emploi à faible volume horaire, à durée déterminée et/ou à salaire faible, on peut en effet craindre que ce dispositif fragilise les perspectives d'insertion à terme des bénéficiaires et substitue une "trappe à précarité " à la " trappe à chômage " créée par le dispositif d'indemnisation. Ces effets de la pratique d'activité réduite et du dispositif d'intéressement de l'Unedic méritent, comme on le voit, d'être établis, tant d'un point de vue théorique qu'empirique. $^{2}$

L'objet du présent article est de procéder à un état des lieux des principales études ayant examiné les effets des dispositifs d'intéressement à l'exercice d'activités transitoires sur le retour à l'emploi des chômeurs.

Après avoir rappelé les principales caractéristiques du dispositif réglementaire en vigueur en France et dans les principaux pays européens, nous procédons à une analyse théorique des effets qu'il est possible d'attendre du dispositif d'intéressement. Nous examinons les effets possibles du dispositif tant sur la décision d'activité à un moment donné du temps, dans un cadre statique, que sur la dynamique de recherche d'emploi. Dans cette première partie, nous discutons alors les possibles canaux de transmission par lesquels pourraient transiter les effets de la mesure d'intéressement. Nous soulevons aussi la question du 
ciblage de la mesure d'intéressement et de sa substituabilité possible à d'autres dispositifs de la politique de l'emploi. Nous procédons ensuite à un examen des difficultés méthodologiques que soulèvent l'évaluation des effets de la politique d'intéressement et plus largement l'analyse de bon nombre de dispositifs de la politique publique. La dernière section est consacrée à une synthèse des principaux travaux empiriques consacrés à l'effet du dispositif d'intéressement et de la pratique d'activité réduite en matière de retour à l'emploi. Enfin nous dressons un bilan des principaux résultats et dégageons les questions majeures qui devraient être abordées par les travaux futurs.

\section{Le dispositif réglementaire}

Avant d'aborder la question de l'évaluation du dispositif d'intéressement des allocataires du chômage, il est utile de rappeler succinctement les modalités et principes d'application du cumul d'un revenu à une allocation de chômage.

\section{Chômeurs du régime général de l'Unedic}

Le dispositif ARAC de l'Unedic permet aux chômeurs indemnisés de cumuler partiellement revenu d'activité et allocation chômage, à condition de rester inscrit en tant que demandeur d'emploi à l'ANPE. Les possibilités de cumul offertes dans le cadre de l'Allocation unique dégressive (AUD) sont maintenues dans le cadre de l'Allocation d'aide au retour à l'emploi (ARE), en place

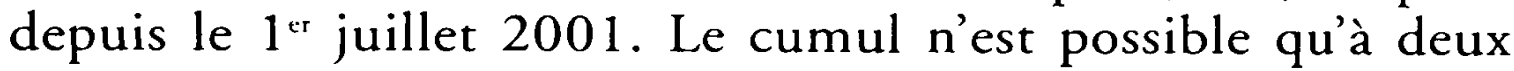
conditions : l'activité exercée ne doit pas excéder une durée de travail mensuelle de 136 heures et la rémunération mensuelle ne doit pas dépasser $70 \%$ du salaire perçu dans l'emploi précédant l'entrée en chômage'. 
Si ces conditions sont vérifiées, l'Assedic calcule alors un nombre de jours non indemnisables dans le mois au cours duquel l'allocataire a travaillé, à partir de la règle suivante : le nombre de jours non indemnisables est défini par le rapport entre le revenu mensuel tiré de l'activité réduite et le salaire journalier de référence (salaire pris en compte pour le calcul des contributions au titre des assurances-chômage durant les douze derniers mois de l'emploi antérieur). Une fois déduits ces jours non indemnisables, l'indemnisation du chômage a lieu dans les mêmes conditions qu'en l'absence d'activité : le taux de remplacement et le salaire de référence pris en compte dans le calcul des indemnités restent les mêmes. Ceci revient à appliquer le taux de remplacement pertinent au salaire mensuel de référence diminué des revenus mensuels de l'activité réduite. Pour les chômeurs de plus de 50 ans, un coefficient de minoration de 0.8 est appliqué au salaire de l'activité réduite dans le calcul des indemnités.

Par ailleurs, les jours non indemnisés repoussent d'autant la durée d'indemnisation à laquelle l'individu peut prétendre, dans la limite de dix-huit mois d'activité réduite ${ }^{\prime}$. Ce report de droit bénéficie aussi aux chômeurs indemnisés dépassant les plafonds de durée de travail ou de rémunération, à condition qu'ils restent inscrits auprès de l'ANPE.

Pendant la période d'intéressement, le revenu disponible d'un chômeur bénéficiant d'un taux de remplacement $q$, qui percevait un salaire horaire $w_{0}$ pour une durée mensuelle de travail $h_{0}$ avant d'entrer en chômage, et exerçant une activité rémunérée au salaire horaire $w_{l}$ pour une durée mensuelle $h_{l}$, sera donc donné par:

$$
R= \begin{cases}q w_{0} h_{0} & \text { en l'absence d'activité } \\ q w_{0} h_{0}+w_{1} h_{1}(1-q) & \text { si } h_{1} \leq 136 \text { et } w_{1} h_{1} \leq 0.7 w_{0} h_{0} \\ w_{l} h_{l} & \text { si } h_{1}>136 \text { ou } w_{1} h_{1}>0.7 w_{0} h_{0}\end{cases}
$$

Pour le bénéficiaire, le dispositif d'intéressement conduit donc à augmenter les ressources monétaires disponibles, tandis que le montant des prestations versées par la caisse d'assurancechômage est réduit. 


\section{Chômeurs bénéficiaires du régime de solidarité de l'Unedic ou de la solidarité nationale}

Des possibilités de cumul d'un revenu d'activité avec les ressources d'allocations existent aussi pour les bénéficiaires de l'Allocation spécifique de solidarité (ASS), de l'Allocation d'insertion (AI) et du Rmi.

Pour le régime de solidarité de l'Unedic, le cumul d'un revenu d'activité et de l'ASS ou l'AI ${ }^{5}$ a été instauré par la loi de lutte contre les exclusions de juillet 1998. Seuls les individus inscrits en tant que demandeurs d'emploi, ou dispensés de recherche d'emploi, peuvent bénéficier de l'intéressement. Pendant les six premiers mois d'activité réduite, si le salaire est inférieur à la moitié du Smic net, l'individu cumule intégralement son revenu et l'allocation de solidarité. Si la condition de rémunération n'est pas vérifiée, l'allocation de base est amputée à hauteur de $50 \%$ de la partie du revenu net excédant la moitié du Smic. A partir du septième mois d'activité réduite, quelque soit le revenu, le montant de l'allocation de base est réduit de la moitié du revenu net d'activité. Le cumul est autorisé pour une période de douze mois.

Dans le cadre du Rmi ou de l'API, l'allocataire qui reprend une activité cumule intégralement revenu et allocation jusqu'à la révision trimestrielle de ses droits à allocation. Après cette révision et pour les trois révisions trimestrielles ultérieures, la moyenne des revenus du trimestre précédent est affectée d'un abattement de $50 \%$ pour le calcul du montant de l'allocation versée : le taux de cumul passe alors de $100 \%$ à $50 \%$.

\section{Réglementations étrangères}

Des dispositifs d'inspiration similaire sont aussi à l'œuvre dans différents pays européens'. Un premier groupe de pays maintient les droits à indemnisation du chômage antérieurement acquis en 
cas de reprise d'activité temporaire, mais interrompt alors le versement de l'allocation. La durée pendant laquelle les droits sont maintenus varie : deux ans au Portugal, un an en Suède, six mois aux Pays-Bas, cinq jours en Italie. Dans d'autres pays, la reprise d'une activité temporaire n'interrompt pas le versement des indemnités mais les sommes versées sont réduites de la valeur des revenus d'activité : il n'y a dans ce cas pas de cumul. C'est le cas au Danemark, en Grèce et en Irlande ${ }^{7}$. Au Royaume-Uni, les revenus d'activité sont déduits du montant de l'indemnité mais la moitié des allocations non perçues est placée sur un compteépargne qui est ensuite versé à l'allocataire en cas de reprise d'emploi définitive.

D'autres pays autorisent un cumul partiel des revenus d'activité et des allocations chômage. En Autriche et en Belgique, le cumul est total jusqu'à un revenu plafond au-delà duquel les indemnités sont suspendues. En Finlande, il est possible de cumuler la moitié des allocations chômage avec un revenu d'activité. En Allemagne, le taux de cumul est variable en fonction des gains d'activité. Il peut atteindre $80 \%$. Pour bénéficier du cumul, la durée de l'activité transitoire ne doit pas dépasser quinze heures par semaine. Enfin, en Suisse, il existe un dispositif très proche du dispositif français, offrant des conditions de cumul similaires ainsi que la possibilité de reporter les droits à indemnisation au-delà de la période d'activité réduite.

\section{Effets attendus et limites du dispositif d'intéressement}

En autorisant le cumul d'une partie des indemnités chômage avec des revenus d'activité, le dispositif d'intéressement de l'Unedic vise implicitement à atténuer les effets désincitatifs de l'assurance-chômage. Par ailleurs, au-delà de ces effets de court-terme, 
un des objectifs souhaités est d'améliorer, à plus longue échéance, les conditions d'insertion au marché du travail des chômeurs.

\section{Diminuer les effets désincitatifs de l'assurance-chômage}

Un des reproches adressés de longue date aux systèmes d'indemnisation du chômage est qu'ils décourageraient le retour à l'emploi des chômeurs en supprimant pour partie les gains à la reprise d'activité. En autorisant un cumul partiel, le système d'intéressement restaurerait les gains à la reprise d'activité. Les possibilités de cumul dans ce dispositif sont cependant différenciées selon le type d'emploi retrouvé et selon le niveau d'indemnisation préalable du chômeur. Il convient alors de s'interroger sur la nature des emplois que les chômeurs seront le plus incités à reprendre et de se demander quelles sous-populations de l'ensemble des chômeurs seront les plus intéressées à reprendre un emploi dans le cadre du dispositif de cumul.

\section{L'effet désincitatif de l'assurance-chômage}

L'effet désincitatif de l'assurance-chômage sur l'offre de travail provient du fait qu'en l'absence d'intéressement, tout exercice d'une activité rémunérée se traduit par la suppression des indemnités chômage, et ce quel que soit le nombre d'heures travaillées. De ce fait, la reprise d'emploi en activité réduite, c'est-à-dire pour une faible durée de travail et/ou un faible niveau de rémunération horaire pourra conduire à une baisse de revenu disponible.

La figure $n^{\circ} 1$ représente le niveau de ressources totales $R$ d'un chômeur indemnisé (en ordonnée) en fonction du volume d'heures travaillées (en abscisse). Avec les notations définies précédemment, le revenu disponible en l'absence d'activité est égal à $B=q w_{0} h_{0}$. On suppose que le chômeur a accès à un emploi versant un salaire horaire net $w_{l}$. En cas de reprise d'activité, le revenu est donné, en fonction du nombre d'heures travaillées, par la droite de pente $w_{l}$ et toute activité se traduit par la perte des indemnités de chômage. Pour un volume d'heures travaillées inférieur 
Figure 1

Contrainte budgétaire en l'absence d'intéressement

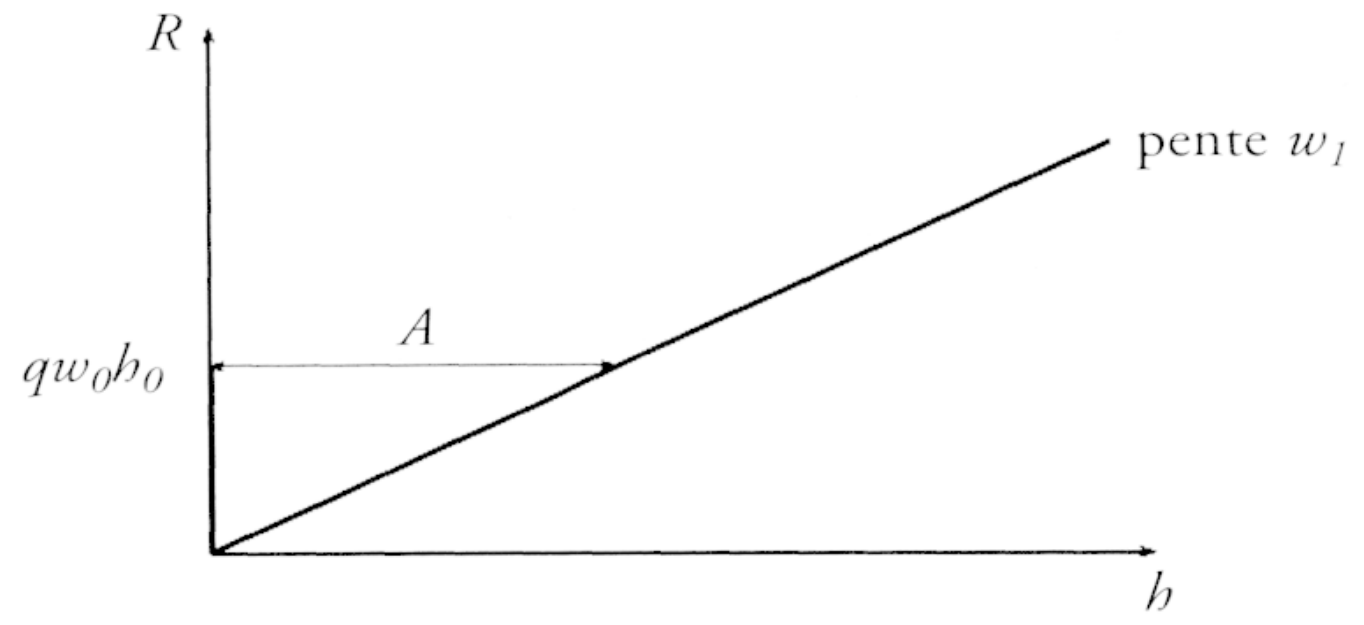

à $B / w_{1}$ (zone $A$ du graphique), les ressources de l'agent sont inférieures lorsqu'il travaille à ce qu'il percevrait en restant au chômage. L'effet désincitatif de l'assurance-chômage correspond donc au fait que, si on raisonne uniquement en termes de revenu monétaire disponible, l'agent préférera strictement rester au chômage plutôt que d'exercer une activité dans la zone $A$. Ces effets désincitatifs seront par ailleurs d'autant plus élevés que le taux d'indemnisation $q$ sera élevé et que le salaire de réembauche auquel peut prétendre le chômeur sera faible, puisque l'étendue de la zone $A$ dépend du ratio $q w_{0} h_{0} / w_{l}$.

Différents éléments suggèrent que ces effets désincitatifs peuvent être quantitativement importants ${ }^{8}$. Les études existantes montrent que les salaires de réembauche des personnes transitant par le chômage sont en général de 10 à $15 \%$ inférieurs aux salaires perçus avant le passage par le chômage (voir Cohen, Lefranc et Saint-Paul [1997] pour le cas français), ce qui conduit à une valeur raisonnable de $w_{0} / w_{1}$ de l'ordre de 1.1 à 1.15 . Ce chiffre doit alors être comparé à la valeur du taux de remplacement net. Celui-ci dépend de la durée de chômage et de la filière d'indemnisation. Selon les cas, il variera entre $83 \%$ pour un salaire de référence égal au $\mathrm{Smic}$ à environ $70 \%$ pour un salaire de référence égal à trois fois le $S_{m i c}$. Pour de telles valeurs du taux de 
remplacement, les gains à la reprise d'emploi peuvent donc s'avérer particulièrement faibles, voire négatifs, même pour des volumes d'heures mensuels assez élevés.

En outre, dans la mesure où, d'une part, la perception de l'allocation chômage peut s'accompagner de la perception d'autres prestations complémentaires et conditionnées aux ressources individuelles ${ }^{10}$ et où, d'autre part, l'exercice d'une activité salariée fait subir des coûts additionnels (transport, garde d'enfant, ...) les effets désincitatifs peuvent être plus élevés encore. Les effets désincitatifs à court terme de l'assurance-chômage seront toutefois moindres dès lors que les chômeurs indemnisés ont une préférence intrinsèque pour l'emploi par rapport à l'état de chômage"'.

\section{L'effet incitatif de l'intéressement}

En autorisant le cumul partiel de l'indemnité chômage et des revenus d'activité, le mécanisme d'intéressement a pour effet d'accroître les incitations à la reprise d'emploi dans la limite des conditions de cumul.

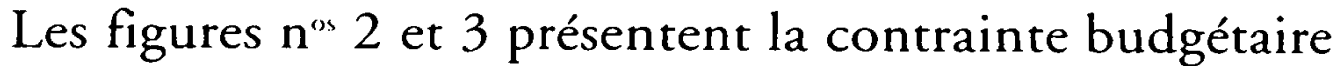
d'un chômeur en fonction des heures travaillées en présence d'intéressement. Sur la première figure, on a supposé que le salaire horaire "offert " est tel qu'un volume horaire de 136 heures mensuelles conduit à une rémunération inférieure à $70 \%$ de la rémunération antérieure. La figure $\mathrm{n}^{\circ} 3$ correspond au cas complémentaire $\left(w_{1} 136>0.7 w_{0} h_{0}\right)$. Sur chacune des figures, la relation entre revenu disponible et heures travaillées est donnée par un premier segment croissant, en trait plein, de pente $(1-q) w_{1}$, suivi d'un segment vertical et d'un troisième segment croissant de pente $w_{l}$.

En comparant les figures $n^{\text {os }} 2$ et 3 à la figure $n^{\circ} 1$, on voit que l'intéressement annule l'effet désincitatif de l'assurancechômage en supprimant la chute initiale du revenu disponible, pour des horaires de travail faibles (zone $A$ de la figure $\mathrm{n}^{\circ} 1$ ). Dans la zone de cumul, le gain net issu de l'exercice d'une activité est donné par le terme $w_{1} h_{1}(1-q)$ dans l'équation (1) et l'intéresse- 
Figure 2

Contrainte budgétaire en présence d'intéressement

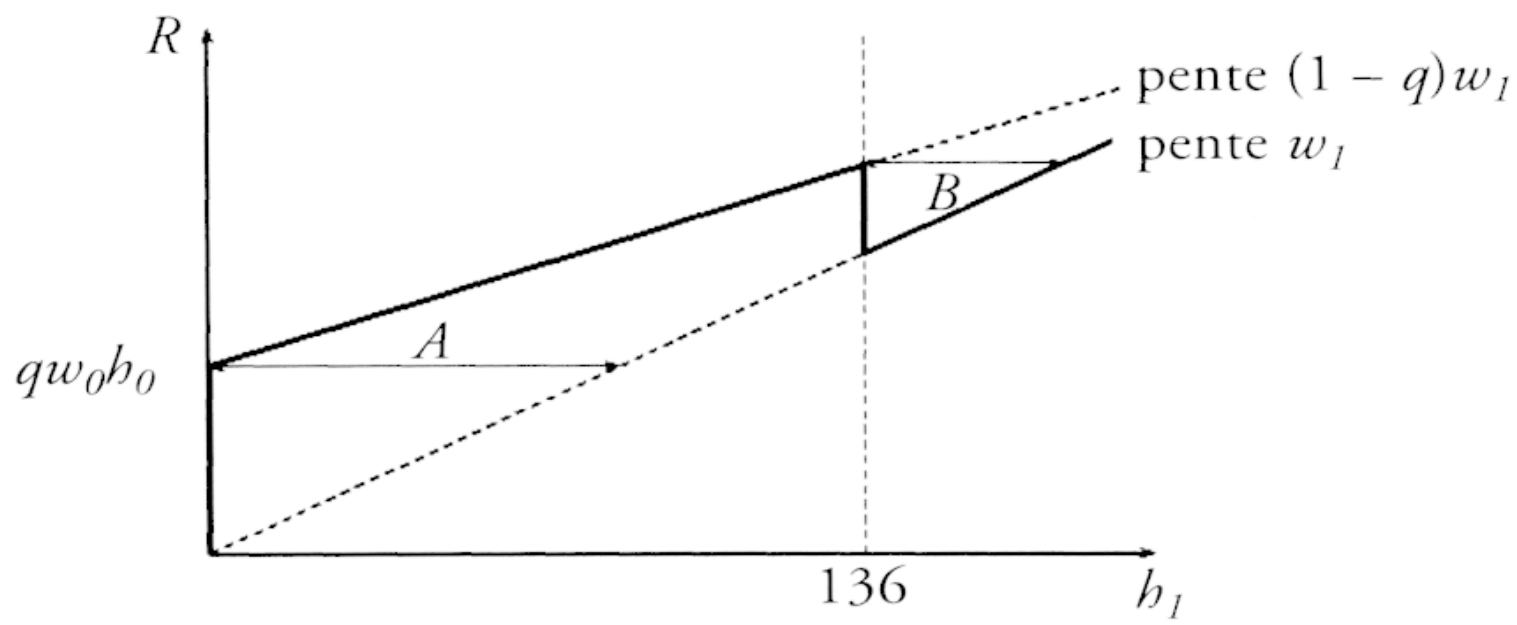

Figure 3

Contrainte budgétaire en présence d'intéressement

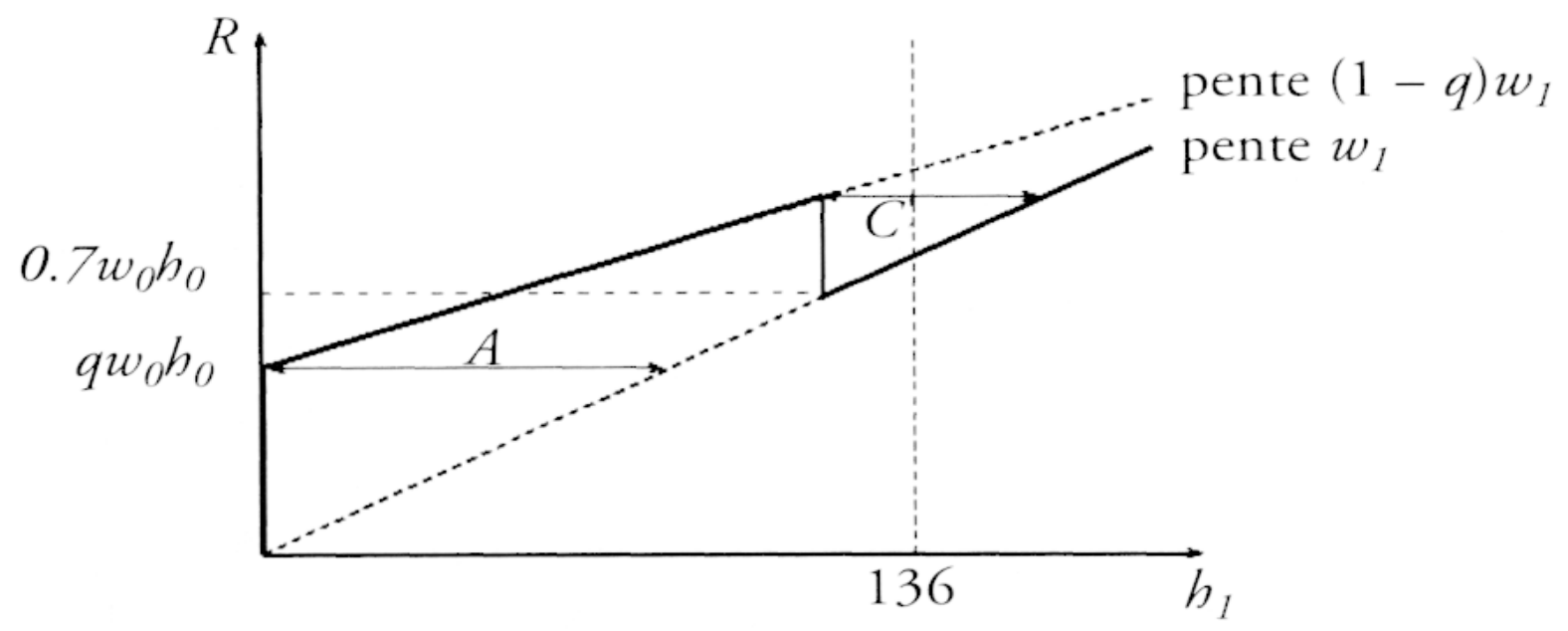

ment s'apparente donc à un maintien des allocations chômage accompagné d'un prélèvement sur les revenus du travail au taux $q$.

Des incitations variables selon les emplois et les conditions d'indemnisation

Les incitations créées par l'ARAC varieront cependant selon la durée du travail et la rémunération dans l'emploi en activité 
réduite. Les conditions d'intéressement créent tout d'abord une nouvelle discontinuité dans le profil revenu-heures travaillées. Cette discontinuité intervient autour d'un nombre d'heures de travail égal à 136 dans la figure $n^{\circ} 2$ et pour un niveau de revenu égal $70 \%$ de la rémunération antérieure dans la figure $\mathrm{n}^{\circ} 3$. Dans chacun des cas, les emplois des zones $B$ ou $C$ conduisent à des ressources totales plus faibles que celles atteintes au point de discontinuité, en dépit d'un volume horaire mensuel plus élevé. Les incitations à accepter des emplois à faible volume de travail horaire ou faible rémunération sont donc renforcées par le fait qu'il n'est pas attractif d'accepter des emplois dans les zones $B$ (figure $\mathrm{n}^{\circ} 2$ ) et $C$ (figure $\mathrm{n}^{\circ} 3$ ), comparativement aux emplois situés à la limite de la zone d'intéressement. Notons d'ailleurs que les combinaisons salaire-heures de ces deux zones procurent un niveau de ressources supérieur au seul montant des indemnités chômage et seraient donc susceptibles d'être acceptées en l'absence d'intéressement.

Par ailleurs, pour un couple salaire-heures travaillées vérifiant les conditions de cumul, le gain monétaire à l'exercice d'une activité réduite $\left((I-q) w_{1} h_{l}\right)$, et donc les incitations créées par le système d'intéressement, varieront en fonction du taux de remplacement ${ }^{12}$. Ce taux de remplacement est lui-même déterminé en fonction de la durée d'affiliation de l'individu, de son niveau de salaire antérieur au chômage et de sa durée de chômage. Toutes choses égales par ailleurs, les incitations à l'activité réduite seront donc d'autant plus faibles que le salaire antérieur était luimême faible. D'autre part, les individus ayant une durée d'affiliation plus faible bénéficieront de l'indemnisation du régime général pendant une période plus courte et entreront plus rapidement dans le régime de solidarité de l'Unedic où les conditions d'intéressement sont plus incitatives ${ }^{13}$. Au total, le dispositif de l'Unedic instaure donc implicitement un ciblage des mesures d'intéressement en faveur des individus à forte durée de chômage, ayant une durée d'affiliation faible et/ou - bien que cet attribut soit souvent antinomique des deux premiers - à salaire élevé. 


\section{Assurer une insertion durable au marché du travail}

Le mécanisme d'intéressement de l'Unedic ne s'apparente cependant pas à une mesure permanente d'incitation financière à la reprise d'activité telle que la prime pour l'emploi. Les possibilités de cumul sont en effet limitées dans le temps et le cumul est conditionnel au fait de rester en recherche d'emploi. Le second objectif, à plus long terme, assigné au dispositif, semble être de favoriser une insertion au marché du travail durable et d'éviter l'apparition d'un chômage de longue durée. Les mécanismes par lesquels pourrait transiter cet effet méritent cependant d'être explicités et mis en perspective.

\section{Augmenter le taux de sortie du chômage et limiter l'étendue du chômage de longue durée}

De nombreuses études ont souligné les faibles probabilités de reprise d'emploi de certaines catégories de chômeurs. Schématiquement, les taux de transition du chômage vers l'emploi apparaissent plus faibles pour les chômeurs les moins qualifiés et pour les individus ayant une plus longue durée de chômage écoulée ${ }^{14}$. Afin de comprendre comment le dispositif d'intéressement peut favoriser la sortie du chômage et limiter le développement du chômage de longue durée, il convient de revenir sur les déterminants individuels des probabilités de transition.

Un des enseignements des modèles de recherche d'emploi est que la probabilité individuelle de sortie du chômage résulte de deux facteurs : d'une part la réception d'offres d'emploi, d'autre part la décision individuelle d'acceptation des offres éventuellement reçues. Les effets bénéfiques attendus du dispositif d'intéressement en termes de taux de sortie du chômage transitent par ces deux composantes.

L'exercice d'une activité réduite pendant la recherche d'emploi est tout d'abord susceptible de favoriser la réception d'offres d'emploi en maintenant les individus en chômage dans un milieu professionnel propice. L'examen des modes de recherche d'emploi conduisant le plus fréquemment à une sortie du chô- 
mage révèle en effet que les relations professionnelles et les contacts directs avec les employeurs contribuent à une fraction importante des retours à l'emploi. Dans le cas de la France, le mode d'obtention de l'emploi actuel peut être étudié à partir de l'enquête Emploi. Le tableau $n^{\circ} 1$, obtenu à partir de l'enquête Emploi de 1999, montre l'importance des relations personnelles et des contacts directs avec les employeurs dans la sortie du chômage : ces deux modes de recherche d'emploi contribuent à la moitié des nouveaux appariements ${ }^{15}$. Ces résultats peuvent être rapprochés de ceux obtenus par Granovetter [1974] dans une étude proche, quoiqu'un peu ancienne et portant sur les EtatsUnis. Il montre que $56 \%$ des individus ont obtenu un emploi par un contact personnel, parmi lesquels $70 \%$ l'ont obtenu par relation professionnelle.

Ce maintien du lien professionnel peut en outre s'avérer particulièrement important pour les chômeurs de longue durée. L'étude de McCormick [1991], menée en 1986 sur un échantillon de chômeurs britanniques, montre en effet un déclin des contacts directs avec les employeurs potentiels, à mesure que la durée de chômage augmente : parmi les chômeurs de moins de six mois, $60 \%$ avaient eu un contact direct avec un employeur dans leurs démarches de recherche d'emploi alors que parmi ceux de plus de six mois de chômage, seuls $40 \%$ avaient eu un contact direct ${ }^{16}$.

Enfin, en augmentant les gains à la reprise d'emploi, le dispositif d'intéressement peut aussi conduire à augmenter l'effort individuel de recherche d'emploi et donc le taux de réception d'offres d'emploi : c'est ce que suggère en partie l'article de McCall [1996] sur lequel nous reviendrons plus loin.

Par ailleurs, le dispositif d'intéressement peut aussi influencer la probabilité d'acceptation des offres reçues par les chômeurs. Les modèles micro-économiques de recherche d'emploi indiquent que la décision d'acceptation des offres d'emploi reçues résulte de la comparaison entre le salaire offert et un seuil d'exigences salariales ou salaire de réservation. Une augmentation de la valeur moyenne des salaires offerts ou une baisse du salaire de réservation conduiront toutes deux à une hausse de la probabi- 
Tableau 1 :

Mode d'obtention de l'emploi occupé

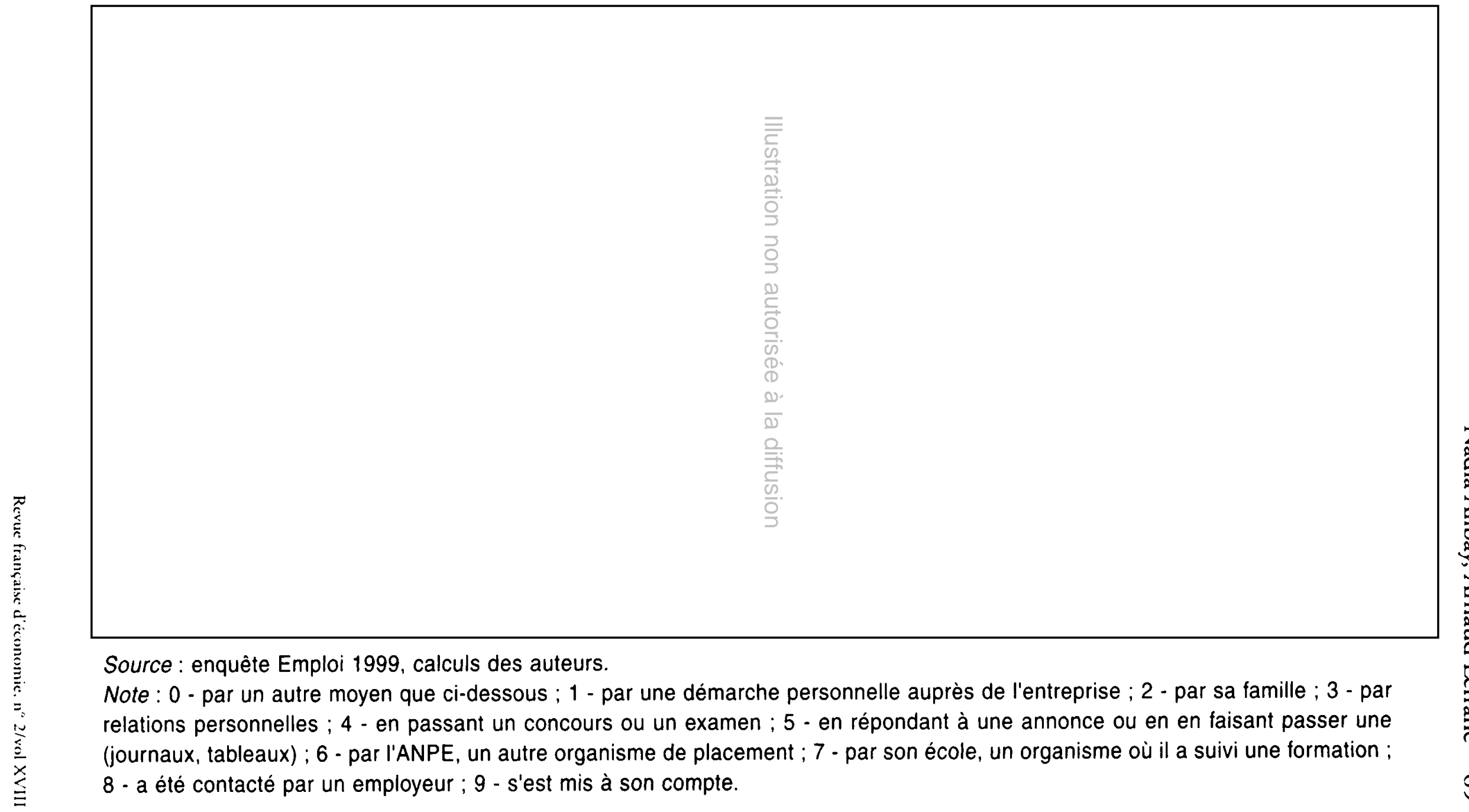


lité qu'une offre d'emploi soit acceptée ${ }^{17}$. Le dispositif d'intéressement peut avoir ces deux effets.

L'effet transitant par la baisse du salaire de réservation n'est que temporaire et résulte de l'augmentation des incitations relatives à la reprise d'emploi, par rapport aux gains en chômage, déjà discutée dans la section précédente. L'effet sur la valeur moyenne des salaires offerts dépend quant à lui de l'incidence des activités réduites sur le niveau de capital humain des chômeurs.

Certains travaux ont suggéré que le maintien en chômage conduirait à une dépréciation du stock de capital humain individuel $^{18}$. Il existe relativement peu d'évaluations empiriques de cette hypothèse, faute d'une mesure claire du niveau de capital humain individuel. Néanmoins, deux types de résultats suggèrent la pertinence empirique de cette conjecture. D'une part, les réponses aux enquêtes auprès d'entreprises, exploitées dans Winter-Ebmer [1991], indiquent que les employeurs considèrent que, toutes choses égales par ailleurs, le niveau de capital humain est plus faible parmi les chômeurs de longue durée. D'autre part, il semble avéré que les salaires de réembauche décroissent avec la durée du chômage, ce qui pourrait aussi indiquer une décroissance de la productivité individuelle avec la durée de chômage ${ }^{19}$.

Dans ce contexte, on peut penser que l'exercice d'activité réduite permettra l'accumulation de capital humain additionnel ou limitera l'étendue des pertes de capital humain liées au chômage. Il pourrait aussi permettre aux chômeurs de signaler une motivation professionnelle particulière aux futurs employeurs. De nombreux travaux valident l'hypothèse beckerienne d'accumulation de capital humain "sur le tas " liée à l'expérience professionnelle. Cet effet variera, à l'évidence, en fonction des caractéristiques de l'emploi occupé. Dans la mesure où le dispositif d'intéressement restreint les possibilités de cumul à des emplois temporaires et à temps partiel, on peut mettre en doute l'intérêt des firmes à s'engager dans un processus de formation des travailleurs bénéficiant de l'intéressement. Toutefois les travaux sur la dynamique des salaires au sein des emplois précaires, notamment Booth et al [2002], révèlent l'existence de rendements significatifs de l'expérience accumulée dans ces emplois. Si tel est 
aussi le cas des emplois occupés en activité réduite, le dispositif d'intéressement, en maintenant ou en accroissant le niveau de capital humain des personnes en recherche d'emploi, aura donc pour effet de favoriser à terme la sortie du chômage.

\section{Un substitut aux autres dispositifs de la politique active du marché du travail?}

On remarquera que les effets potentiels du dispositif d'intéressement sur la probabilité de retour à l'emploi recoupent à la fois ceux attendus des dispositifs d'aide au placement et à la recherche d'emploi, et ceux des programmes de formation. Il convient donc de s'interroger sur la substituabilité éventuelle du dispositif d'intéressement à d'autres programmes de la politique active. Ceci suppose une comparaison des effets, des coûts et des populations bénéficiaires des différents dispositifs, qui dépasse largement le cadre de cet article et on renvoie à Heckman et al. [1999] pour une discussion approfondie des effets de politiques actives en Europe et aux Etats-Unis ${ }^{20}$.

Toutefois, il est probable que la place du dispositif d'intéressement dans la politique de l'emploi ne se réduise pas, en France au moins, à cette éventuelle substituabilité. La participation au dispositif d'intéressement n'est en particulier pas exclusive de la participation à différents programmes de formation en emploi : les bénéficiaires de contrats emploi-solidarité peuvent par exemple cumuler revenu d'activité et allocation chômage au titre de I'ARAC. De ce point de vue, la dimension incitative du dispositif d'intéressement permettrait donc de soutenir le développement d'autres dispositifs de la politique active.

Il convient aussi de s'interroger sur l'efficacité du ciblage du dispositif d'intéressement au regard des effets attendus sur le retour à l'emploi. On a déjà noté que les incitations créées par l'ARAC étaient plus faibles pour les personnes bénéficiant de taux de remplacement plus élevés, notamment les travailleurs les moins qualifiés et ceux ayant une durée d'affiliation importante. Or cette population est précisément celle qui connaît les plus fortes difficultés de retour à l'emploi et qui bénéficierait vraisembla- 
blement le plus des effets positifs décrits ci-dessus. Le ciblage implicite de l'ARAC apparaît de ce point de vue en partie inadéquat.

On a aussi remarqué que les incitations à l'activité réduite augmentaient avec la durée de chômage. Un tel ciblage sur les chômeurs de longue durée peut, là encore, sembler en partie inadéquat. Pour le comprendre, il convient de revenir sur les causes du chômage de longue durée. La plupart des travaux micro-économétriques montrent sur ce point que le taux de retour à l'emploi décroît en moyenne avec la durée de chômage. Cet effet apparent de la durée de chômage peut cependant refléter deux causes distinctes. D'une part un effet propre de la durée de chômage sur le taux de sortie individuel. D'autre part, l'existence d'une hétérogénéité individuelle des taux de sortie du chômage conduisant, même en l'absence d'effet propre de la durée de chômage, à une décroissance du taux de sortie moyen, du fait d'un effet de composition ${ }^{21}$. Si un ciblage du dispositif sur les chômeurs de longue durée apparaît souhaitable dans le premier cas, il serait préférable, dans le second cas, de concentrer dès le début de l'épisode de chômage les incitations sur les individus les plus fragiles en matière d'insertion.

Or, les études empiriques existantes suggèrent que l'effet observé de la durée de chômage sur le taux de sortie tient en grande partie à l'existence d'hétérogénéité individuelle. Bonnal, Fougère et Sérandon [1997] trouvent qu'après prise en compte de l'hétérogénéité individuelle, il n'y a de dépendance du taux de sortie du chômage à la durée écoulée que pour les jeunes les moins éduqués ${ }^{22}$. L'article récent de Van den Berg et al. [2000] apporte des résultats plus larges. Il montre qu'au cours des quatre premiers trimestres de recherche d'emploi, il n'existe pas de dépendance du taux de sortie à la durée écoulée pour les plus jeunes et une dépendance (légèrement) positive pour les autres types de chômeurs. En revanche, à partir de quatre trimestres de recherche d'emploi, ils trouvent une dépendance négative et significative à la durée écoulée. Au vu de ces résultats, le ciblage implicite des incitations sur les chômeurs de longue durée apparaît là encore mal adapté. 


\section{Favoriser l'insertion dans des emplois stables}

L'analyse des taux de transition du chômage vers l'emploi, considéré comme un état homogène, ne suffit pas à appréhender les effets en termes d'insertion du dispositif d'intéressement. Il convient en effet d'examiner aussi dans quelle mesure l'exercice d'activité réduite influence le type d'emploi retrouvé à la sortie du chômage et l'éventuelle récurrence du chômage.

Un des présupposés au développement des possibilités de cumul est que l'exercice d'activité réduite améliorera, à terme, les conditions d'insertion au marché du travail des chômeurs, en augmentant, par exemple, les chances de sortir du chômage vers un emploi stable ou la qualité de l'emploi occupé à la sortie du chômage. A cette vertu potentielle du dispositif d'intéressement sur l'insertion, on pourrait tout aussi bien objecter la possibilité qu'il conduise à une précarisation des situations individuelles sur le marché du travail, menant à terme à des emplois instables et à une récurrence accrue du chômage. Ces effets mériteraient d'être examinés empiriquement dans le cadre des travaux d'évaluation du dispositif ARAC. Les travaux existants sur les transitions entre emploi précaires et emplois stables permettent d'apporter quelques éléments de réponse préliminaires.

La question de l'effet de l'exercice d'emplois d'attente sur l'insertion à terme a été principalement abordée du point de vue des transitions entre emplois stables et emplois précaires. Dans les études françaises, cette distinction est, en général, appréhendée à partir de la distinction entre emplois à durée déterminée (CDD, intérim) et emplois à durée indéterminée (CDI). Les premiers travaux de Fougère et Kamionka [1992b] portant sur des données de la fin des années 1980 , indiquent une probabilité de transition annuelle assez forte entre emploi précaire et emploi non-précaire : la probabilité moyenne de transition annuelle entre emploi précaire et emploi non-précaire estimée dans cet article est de l'ordre de 3/4 pour les hommes et $2 / 3$ pour les femmes. En outre, la probabilité de transition vers l'emploi stable à partir d'un emploi précaire est en général plus élevée qu’à partir de l'état de chômage. Cette relative ouverture des emplois pré- 
caires vers des emplois plus stables est aussi notée dans les travaux portant sur d'autres pays, notamment Booth et al [2002]. Au regard de ces résultats, on peut donc attendre du dispositif d'intéressement des effets favorables en matière d'accès aux emplois stables.

Dans le cas de la France, les travaux plus récents de Blanchard et Landier [2002] indiquent cependant, pour des travailleurs jeunes, un net ralentissement des transitions entre CDD et CDI depuis 1990. Par ailleurs, les travaux complémentaires de Fougère et Kamionka [1992a] indiquent la présence d'une hétérogénéité entre individus des taux de transition de l'emploi stable vers l'emploi précaire. Derrière des taux de transition moyens élevés, il existerait dans certains segments du marché du travail une proportion importante (jusqu'à $20 \%$ ) d'individus cantonnés à une succession d'emplois précaires et d'épisodes de chômage. Magnac [2000] conclut aussi à l'existence d'une hétérogénéité inobservable significative, dans le cas des travailleurs les plus jeunes. En outre, sur ce segment du marché du travail, une fois prise en compte l'hétérogénéité inobservable, le fait d'occuper un emploi précaire, ne semble pas augmenter les chances de trouver un emploi stable par rapport à l'état de chômage.

Ces résultats laissent en grande partie inexpliquée l'origine de l'hétérogénéité des taux de transitions individuels. On peut craindre, comme le notent plusieurs auteurs, que cette hétérogénéité apparente soit le produit des trajectoires passées sur le marché du travail et que les difficultés de retour à l'emploi stable proviennent de l'effet persistant d'expériences passées d'emploi précaire.

Cette hypothèse d'un effet persistant des trajectoires passées sur les perspectives futures d'insertion au marché du travail a fait l'objet de plusieurs études empiriques, aboutissant parfois à des résultats opposés. La plupart des études se confinent dans l'examen des transitions entre le chômage et l'emploi, considéré comme un état homogène. Les travaux séminaux d'Heckman et Borjas [1980] concluent, à partir d'un échantillon restreint de travailleurs américains jeunes, à l'absence d'effet persistant des trajectoires passées. Dans le cas du marché du travail français, Lol- 
livier [2000] montre que l'existence d'un effet positif du nombre d'épisodes de chômage passés sur la probabilité de sortie du chômage s'explique entièrement par la présence d'hétérogénéité individuelle $^{23}$. En revanche, dans le cadre d'un modèle plus riche, tenant compte de plusieurs états d'emploi (emploi stable, emploi précaire, dispositifs de formation), Magnac [2000] conclut à un effet persistant des états passés sur les probabilités de transition ultérieures, même après prise en compte de l'hétérogénéité individuelle inobservable.

On retiendra donc la possibilité qu'une telle persistance vienne limiter les vertus du dispositif d'intéressement en matière d'insertion. En outre, on notera une limite possible du dispositif en présence d'hétérogénéité des taux de transition individuels : il est possible, dans ce cas, que le dispositif bénéficie principalement à des individus connaissant une forte mobilité entre chômage et emplois précaires et qui auraient connu de telles trajectoires même en l'absence d'intéressement, sans que l'intéressement mène pour autant à une intégration effective au marché du travail.

Pour finir, au-delà de l'étude des taux de transition entre emplois stables et précaires, il faut aussi remarquer que le dispositif d'intéressement pourra induire une modification du comportement de recherche d'emploi défavorable à une sortie vers l'emploi stable.

D'une part, dès lors que les modes de recherche different selon les caractéristiques de l'emploi recherché, le mécanisme d'intéressement induira une substitution, dans les efforts de recherche d'emploi, en faveur des emplois vérifiant les conditions de cumul et en défaveur des emplois stables, de longue durée ou à salaire plus élevé. Cet effet de substitution est par exemple analysé dans McCall [1996].

D'autre part, il est aussi possible que le temps consacré au travail en activité réduite se substitue au temps consacré à la recherche d'emploi et diminue ainsi les chances d'accès à un emploi stable. Compte tenu du temps consacré en pratique par les chômeurs à la recherche d'emploi, il est cependant peu vraisemblable que de tels effets soient empiriquement importants ${ }^{24}$ 
mais l'activité réduite pourrait aussi limiter la disponibilité des personnes en recherche d'emploi, par exemple, à se rendre à des entretiens d'embauche.

En dépit de taux de transition assez élevés entre emplois précaires et emplois stables, l'existence d'hétérogénéité individuelle, l'effet persistant des trajectoires précaires et l'ajustement endogène des comportements individuels de recherche d'emploi sont donc susceptibles de limiter les vertus du dispositif d'intéressement en matière d'insertion.

\section{Evaluation des effets de l'intéressement : questions et problèmes méthodologiques}

\section{Quels effets évaluer?}

La discussion des effets attendus du mécanisme d'intéressement indique que les évaluations du dispositif devraient aborder les questions suivantes, portant à la fois sur les effets réels de la mesure d'intéressement et sur ses modes opératoires :

- E1 : l'intéressement favorise-t-il la sortie du chômage ?

- E2 : l'intéressement améliore-t-il l'insertion au marché du travail des chômeurs?

- M1 : par quels mécanismes les effets de l'intéressement transitent-ils?

La question E1 comporte deux volets. Il s'agit d'abord d'examiner dans quelle mesure le dispositif d'intéressement incite effectivement les chômeurs à l'exercice d'une activité réduite temporaire. Cette question peut être étudiée en comparant le recours à ce type d'emploi, selon les conditions dans lesquelles les chômeurs bénéficient de l'intéressement.

Par ailleurs, il s'agit aussi d'évaluer les effets de l'exercice d'une activité réduite temporaire sur les chances de sortie du 
chômage, vers un emploi ne relevant pas du dispositif de cumul. Pour mettre en évidence ces effets, il importerait de modéliser le processus de sortie du chômage et de comparer ces probabilités de sortie en fonction de l'exercice d'une activité réduite antérieure.

La question E2 recoupe en partie celle de l'effet de l'activité réduite sur la sortie du chômage mais elle ne s'y réduit pas. Il s'agit ici, plus globalement, des effets à long terme de l'intéressement sur le taux d'emploi des chômeurs. Or, les perspectives d'emploi à long terme des chômeurs dépendent à la fois des chances qu'ils ont de sortir du chômage vers l'emploi et de la probabilité de ne pas retomber en chômage ultérieurement.

Au-delà de la mise en évidence des effets de l'intéressement, il faut aussi s'interroger sur les mécanismes par lesquels transitent les effets de l'intéressement. Dans la section précédente, on a suggéré un certain nombre de mécanismes possibles (efficacité de la recherche d'emploi, capital humain, discrimination). Il est parfois difficile de disposer de mesures observables de ces différents facteurs de sortie du chômage. Une approche intéressante consisterait cependant à mettre à jour à la fois les caractéristiques des emplois exercés dans le cadre du dispositif d'intéressement et les effets de ce dispositif sur le comportement de recherche d'emploi.

\section{Problèmes méthodologiques}

Pour analyser les effets d'un dispositif, on souhaite comparer la situation d'individus en présence de la mesure évaluée (ici la sortie du chômage) à la situation qui serait la leur en son absence. Comme dans toute analyse quantitative, en économie il n'est jamais possible d'examiner les mêmes individus à la fois dans une situation où ils seraient soumis à la mesure et dans une situation où ils n'y seraient pas soumis. Le problème central est alors de déterminer un groupe de référence, équivalent en tous points au groupe bénéficiant de la mesure évaluée, sauf en ce qui concerne l'accès à cette mesure. Ce groupe de référence permettra alors, 
par différence, d'inférer les effets de la mesure. Les effets estimés de la mesure dépendront donc de façon critique de la pertinence du groupe de référence choisi.

Supposons par exemple qu'on souhaite estimer l'effet du dispositif d'intéressement sur la probabilité de chômage des individus. On souhaiterait pouvoir identifier deux groupes de personnes entrant au chômage et identiques sauf en ceci que le premier, noté $T$, se verrait offrir la possibilité de cumul partiel présentée précédemment, alors que le second, noté $C$, se verrait privé de cette possibilité. Si $\mathbf{u}_{\mathrm{i}}$ désigne le taux de chômage du groupe $i$, alors l'effet de l'intéressement sur le taux de chômage peut se calculer comme la différence $u_{7}-u_{C}$.

De la même façon, on pourrait chercher à expliquer l'effet de l'intéressement sur le niveau de salaire, une fois les individus sortis du chômage. En notant $\mathrm{w}_{\mathrm{i}}$ le niveau de salaire individuel et $T_{i}$ une variable binaire indiquant l'appartenance au groupe de traitement $T\left(T_{i}=1\right)$ ou au groupe de contrôle $C$ $\left(T_{i}=0\right)$, on pourrait évaluer l'effet de la mesure d'intéressement en estimant le modèle :

$$
w_{i}=\bar{w}+\beta T_{i}+\epsilon_{i}
$$

où $\epsilon_{i}$ représente toutes les différences inter-individuelles de salaire qui ne s'expliquent pas par la participation ou non au dispositif évalué. Dans ce cas, le coefficient $\beta$ mesurerait l'effet de l'intéressement sur le salaire individuel.

On notera que pour que cette démarche soit valide, il importe que la seule différence entre les deux groupes $T$ et $C$ soit l'accès à la mesure. Un problème commun à beaucoup de travaux d'estimations tient au fait que, dans la réalité, la participation, voire l'éligibilité à la mesure évaluée, ne sont en général pas indépendantes des caractéristiques individuelles, lesquelles caractéristiques ont par ailleurs un effet indépendant sur la situation individuelle. On parle alors de biais de sélection : les individus qui participent à la mesure évaluée ne constituent pas un échantillon aléatoire de l'ensemble des individus. 
Ainsi, si le groupe $C$ n'a pas les mêmes caractéristiques individuelles que le groupe $T$, il est possible que le taux de chômage $u_{C}$ ne mesure pas convenablement le taux de chômage qui aurait été celui du groupe $T$ en l'absence de mesure d'intéressement. Dans ce cas, $u_{T}-u_{C}$ constituera une estimation biaisée des effets de la mesure.

Le problème de sélection intervient à plusieurs niveaux dans l'évaluation des effets du dispositif d'intéressement.

On notera tout d'abord, en amont du problème de biais de sélection que, du fait que le dispositif d'intéressement est, en France, une mesure relativement ancienne et d'application universelle, il n'est pas possible, parmi les chômeurs indemnisés, de séparer deux groupes dont l'un aurait accès à la mesure d'intéressement et l'autre non. On peut tout au plus distinguer des groupes ayant, du fait de la réglementation, des conditions de cumul différentes. Mais tous seront en principe soumis à la même législation, contrairement aux cas simples considérés dans les exemples ci-dessus. On sera alors amené implicitement à comparer des groupes ayant des incitations différenciées au dispositif d'intéressement.

On voit immédiatement apparaître une première source de biais de sélection dans le fait que les conditions d'accès au dispositif d'intéressement sont déterminées par l'histoire passée de l'individu sur le marché du travail, notamment sa durée d'emploi, son niveau de salaire antérieurement au chômage et sa durée de chômage écoulée dans l'épisode étudié. Il est par exemple probable qu'une partie des individus ayant les conditions d'indemnisation les moins favorables proviennent de façon disproportionnée de groupes d'individus ayant des durées d'affiliation au régime d'assurance plus brèves, elles-mêmes explicables par des difficultés particulières d'insertion au marché du travail. Par ailleurs, on a vu qu'un chômeur indemnisé sera d'autant plus incité à reprendre une activité que son taux de remplacement est faible, c'est-à-dire, toutes choses égales par ailleurs, que le temps passé au chômage est important. Or, il est possible que les individus ayant une ancienneté de chômage importante aient aussi un taux de sortie du chômage plus faible. Si on évalue l'effet de l'in- 
téressement sur la sortie du chômage en comparant simplement les taux de sortie du chômage des individus selon qu'ils ont ou non bénéficié de l'intéressement, mais sans tenir compte de l'ancienneté de chômage ou des différences intrinsèques de taux de sortie entre individus, on risque de sous-estimer l'effet de l'intéressement.

Une seconde source de biais de sélection provient du fait que, même si l'accès à la mesure était assigné de façon aléatoire et indépendante des perspectives futures d'insertion au marché du travail ${ }^{25}$, le recours effectif à la mesure serait vraisemblablement fonction de caractéristiques individuelles influençant les perspectives d'insertion ultérieures ${ }^{26}$.

Supposons par exemple qu'on cherche à isoler l'effet de l'exercice d'une activité réduite sur la probabilité de trouver un emploi, une fois passée la période de cumul. Supposons par ailleurs qu'on ait isolé deux groupes dont l'un se voit accorder la possibilité de cumul partiel et dont l'autre n'a pas accès à la mesure. On pourrait alors calculer l'effet de la mesure, en matière de taux de chômage, en procédant comme dans l'exemple ci-dessus.

Il se trouverait cependant qu'au sein de la population s'étant vu accorder la possibilité de cumul, certains individus auraient effectivement exercé une activité réduite et d'autres pas. On pourrait souhaiter évaluer non pas l'effet moyen mais l'effet de la mesure sur ceux qui ont exercé une activité réduite. On se heurterait alors à une seconde source de biais de sélection dans la mesure où on ne peut a priori penser que ceux qui ont exercé une activité réduite ont en moyenne les mêmes caractéristiques que ceux qui n'ont pas exercé d'activité réduite ou que ceux qui ne se seraient pas vu offrir cette possibilité.

De même, une fois le traitement assigné, c'est-à-dire après l'exercice de l'activité réduite, le statut de sortie n'est pas assigné de façon exogène : par exemple, une fois qu'un certain nombre d'individus auront choisi l'intéressement, certains vont rester dans l'intéressement, d'autres vont le quitter pour passer vers le chômage, d'autres encore vont le quitter pour un emploi stable : là encore, on ne peut considérer que ces différentes popu- 
lations sont comparables au sens où elles auraient des caractéristiques pertinentes identiques quant à leur insertion future au marché du travail.

Au total, il apparaît que l'évaluation des effets du dispositif d'intéressement nécessite une prise en compte détaillée des possibles sources d'hétérogénéité entre la population soumise à l'intéressement, la population profitant effectivement des possibilités de cumul et la population excluse ou ne profitant pas de l'intéressement. La non-prise en compte de cette hétérogénéité, dans des évaluations quantitatives qui reposeront toujours sur une comparaison implicite de populations affectées à des degrés divers par la mesure, risquerait en effet de conduire à une évaluation biaisée des effets du dispositif.

\section{Résultats des études existantes}

Certaines études permettent de répondre aux questions soulevées dans la section précédente, sur les effets du dispositif de cumul allocation-revenu d'activité. Elles peuvent être complétées par l'examen de dispositifs proches, à l'œuvre dans d'autres pays. Après avoir décrit les principales caractéristiques des bénéficiaires et des emplois exercés dans le cadre des mesures d'intéressement, on présentera les principaux résultats concernant les effets incitatifs du dispositif d'intéressement. Enfin on discutera les travaux consacrés à l'effet de la pratique d'une activité réduite sur la sortie du chômage et sur les conditions d'insertion au marché du travail.

\section{Analyse descriptive des pratiques d'activité réduite}

L'article de Bel et al [1998] permet tout d'abord de quantifier l'étendue de l'exercice d'activités réduites. En 1998, parmi l'ensemble des personnes inscrites auprès de l'ANPE déclarant cher- 
cher un emploi à durée indéterminée et à temps plein (DEFM de catégories 1 et 6 ) environ $15 \%$ avaient exercé une activité de plus de 78 heures au cours du mois précédent. Parmi les chômeurs ayant une ancienneté de chômage de plus de 6 mois, $30 \%$ avaient exercé une activité réduite de plus de 78 heures au cours de l'épisode de chômage en cours, cette fraction étant plus élevée à mesure que la durée de chômage augmente. L'activité réduite au sens de l'ANPE étudiée dans cet article ne correspond pas à la définition qu'en donne l'Unedic dans le cadre de l'intéressement. La catégorie 6 de l'ANPE correspond à des personnes ayant travaillé un nombre d'heures au moins égal à 78 heures par mois et n'inclut donc pas l'ensemble des personnes indemnisées en activité réduite. A contrario, le champ d'inscription à l'ANPE dépasse celui de l'indemnisation en chômage.

Les effectifs et caractéristiques des bénéficiaires du dispositif d'activité réduite de l'Unedic sont documentés dans la revue trimestrielle de l'Unedic ${ }^{27}$. Entre 1991 et 2000, le nombre d'allocataires de l'assurance-chômage ayant une activité réduite a plus que triplé en passant de 105800 à 340000 . Ce qui correspond en décembre 2000 à $20 \%$ des allocataires de l'assurance-chômage. $66 \%$ de ces allocataires ont entre 25 et 49 ans, et plus d'un tiers ont pour motif d'inscription à l'Assedic la fin d'une mission d'intérim ou la fin d'un contrat à durée déterminée. De plus, ils sont en majorité bénéficiaires de durées d'indemnisations relativement longues. On notera par ailleurs que, parmi les bénéficiaires potentiels de l'assurance-chômage, un nombre de personnes équivalent exerce une activité dépassant les limites de cumul et se trouve en situation de décalage complet.

Les données de l'Unedic permettent aussi de préciser à quel moment se fait l'entrée dans les dispositifs d'activité réduite. Elles révèlent qu'en moyenne l'entrée a lieu au terme de trois à quatre mois d'indemnisation. Il serait cependant intéressant de disposer de données plus complètes sur la distribution des mois d'entrée en activité réduite : dans la mesure où on peut penser que la distribution de ces mois d'entrée n'est pas symétrique, il 
est probable que la date médiane d'entrée en intéressement soit différente de cette moyenne. Si cette distribution est de même nature que la distribution des durées de chômage, la durée médiane sera plus faible que la durée moyenne.

Les données administratives ne fournissent qu'une information limitée sur les caractéristiques socio-démographiques des bénéficiaires de l'intéressement et sur les caractéristiques des emplois exercés en intéressement. Pour enrichir ces données, Gurgand [2000] procède à l'appariement des données administratives avec des données de l'enquête Trajectoire des demandeurs d'emploi (TDE) de la DARES.

Du fait de la procédure d'enquête, cet appariement n'identifie les caractéristiques des épisodes d'activité réduite que pour un sous-échantillon - vraisemblablement imparfaitement représentatif - des épisodes d'activité réduite observables dans les fichiers administratifs ${ }^{24}$. Il permet néanmoins une première caractérisation de ces emplois.

Il apparaît tout d'abord que la forme dominante d'exercice d'une activité réduite dans le cadre du dispositif d'intéressement est celle de l'emploi en intérim ( $41 \%$ des emplois en activité réduite) ou en CDD (36\%). La forte représentation des emplois en intérim peut, en théorie, trouver deux explications. La première serait que le fait d'exercer une activité réduite conduirait à cette forme d'emploi. La seconde serait que les individus qui exercent des emplois en activité réduite seraient, toutes choses égales par ailleurs, plus enclins à recourir à ces formes d'emploi. Pour distinguer ces deux interprétations, Gurgand examine le type d'emploi occupé hors activité réduite par les individus ayant par ailleurs occupé des emplois en activité réduite au cours de leurs épisodes de chômage. Pour ces derniers emplois, la proportion d'emplois en intérim n'est alors que de $24 \%$, ce qui indique que l'exercice d'une activité réduite modifie en faveur de l'intérim le type d'emploi accepté. Sur la base d'une analyse identique, on notera aussi que l'exercice d'une activité réduite favorise le recours à un contrat aidé : $8.7 \%$ des emplois en activité réduite sont de cette nature alors que pour l'ensemble des emplois exercés hors 
activité réduite par les personnes recourant à l'intéressement la proportion d'emplois aidés est seulement de $6 \%$.

L'étude révèle aussi que les emplois exercés en activité réduite ont en général une durée plus brève et offrent des salaires mensuels plus faibles. Ces salaires mensuels plus faibles s'expliquent eux-mêmes par un volume horaire mensuel plus faible et non pas par un taux de salaire horaire différent. La comparaison des taux de salaire à partir des données de l'enquête TDE suggère des distributions assez proches avec une valeur médiane voisine du Smic.

Les estimations menées dans l'article de Granier et Joutard [1999] mettent aussi en évidence l'influence des caractéristiques socio-démographiques et du type d'emploi recherché sur l'exercice d'une activité réduite, définie à partir des catégories de l'ANPE. Elles révèlent que la pratique d'une activité réduite est plutôt le fait de chômeurs relativement jeunes et à niveau de formation ou de qualification élevé ou intermédiaire. Du fait de l'élévation moyenne du niveau de qualification et de formation depuis un demi-siècle, la non prise en compte d'interactions entre le niveau de formation et l'âge de l'individu ne permet cependant pas, selon toute vraisemblance, de distinguer l'effet propre de la qualification de celui de l'âge.

L'article identifie aussi les secteurs d'activité où l'activité réduite est la plus fréquente. Dans l'ensemble, la pratique d'une activité réduite est plus fréquente dans les secteurs des transports et de l'industrie et plus rare dans les secteurs de la gestion et de la distribution. Dans le cas des hommes elle est aussi plus fréquente dans les secteurs de la santé et des BTP et chez les femmes dans ceux de l'hôtellerie et des services.

Le motif d'inscription au chômage exerce une influence discriminante sur la probabilité d'exercer une activité réduite, au moins au début de l'épisode de chômage. On remarque que les primo-entrants et les personnes ayant connu un CDD avant leur période de chômage ont une probabilité plus forte d'exercer un emploi en activité réduite que les personnes entrées en chômage à la suite d'un licenciement ou d'une démission. 


\section{Effets de l'intéressement sur la pratique d'activité réduite}

\section{Gurgand [2001]}

L'article de Gurgand [2001] analyse l'existence d'un effet incitatif du système d'intéressement. Comme on l'a montré dans la section précédente, les possibilités de cumul partiel, dans le cadre du dispositif de l'Unedic, sont d'autant plus avantageuses que le taux d'indemnisation du chômage est faible. L'étude de Gurgand estime donc l'ampleur de cet effet, afin de déterminer si les individus en recherche d'emploi répondent effectivement aux incitations créées par le dispositif.

Le modèle estimé est un modèle qualitatif dans lequel la probabilité d'exercer un emploi en intéressement dépend du taux de remplacement individuel. Si une diminution du taux de remplacement augmente, en théorie, les incitations aux situations de cumul avec décalage partiel des droits assurantiels, par rapport à la situation d'indemnisation pure, elle affectera aussi les incitations à exercer des emplois qui placent l'individu indemnisé en situation de décalage complet de ses droits assurantiels. Pour neutraliser ce second effet, Gurgand mesure alors la probabilité pour un individu d'être en décalage partiel conditionnellement au fait de ne pas être en décalage complet. L'effet de l'intéressement est estimé au sein d'un modèle logit, dans lequel est incluse une série de variables indicatrices correspondant à différents niveaux du taux de remplacement. Il importe d'inclure la durée d'indemnisation parmi la liste de variables de contrôle. En effet, dans la mesure où le taux de remplacement dépend de la durée d'indemnisation, le fait de ne pas tenir compte de cette durée biaiserait l'estimation de l'effet du taux de remplacement sur la probabilité d'activité réduite ${ }^{22}$.

En outre, pour les raisons présentées plus haut, le taux de remplacement variera en fonction de caractéristiques individuelles qui affecteront aussi de façon directe la probabilité d'exercer une activité réduite. Il importe alors de tenir compte de ces différents facteurs d'hétérogénéité individuelle pour obtenir une 
évaluation de l'effet spécifique du taux de remplacement. A cette fin, le modèle logit estimé inclut un effet fixe individuel qui permet de tenir compte de différences inter-individuelles permanentes dans la propension à exercer une activité réduite. Une fois prise en compte cette hétérogénéité, la mesure de l'effet du taux d'indemnisation sur la probabilité d'activité réduite repose uniquement sur les variations du taux de remplacement au sein d'une ouverture de droits.

L'étude a été réalisée sur un extrait du fichier national des Assedic concernant la cohorte de chômeurs suivis par l'enquête Trajectoire de demandeurs d'emploi ${ }^{30}$.

L'estimation révèle un effet négatif et fortement significatif du niveau du taux de remplacement sur la probabilité d'exercer une activité réduite. L'introduction de la durée passée au chômage et des effets fixes pour chaque ouverture de droits n'influe pas sur le sens et la significativité du taux de remplacement. Enfin, l'introduction d'une variable indicatrice de l'exercice antérieur d'une activité réduite indique une forte corrélation positive entre la probabilité d'exercer une activité réduite et l'exercice passé d'une telle activité.

Pour faciliter la lisibilité des résultats obtenus, l'auteur réalise des simulations pour évaluer l'effet de la variation du taux de remplacement sur la probabilité de cumul : une diminution du taux de remplacement de $10 \%$ augmenterait de 10 à $20 \%$ le nombre de mois en cumul ${ }^{3 !}$.

L'interprétation de cet effet négatif de $q$ sur la probabilité d'exercer une activité réduite reste cependant délicate. L'analyse développée dans l'article indique que l'effet de $q$ provient d'une augmentation des gains monétaires (marginaux) à mesure que le taux de remplacement diminue. L'effet serait alors le suivant : les chômeurs indemnisés intensifieraient leur pratique d'activité réduite car la rémunération de chaque heure travaillée augmente.

Cependant une variation de $q$ affectera aussi la valeur des indemnités perçues en l'absence d'intéressement ou d'activité réduite. Cette baisse des ressources de l'agent aura alors potentiellement un effet incitatif qui s'interprète différemment : 
les chômeurs intensifieraient leur pratique d'activité réduite du fait d'un niveau de ressources trop faible.

La distinction entre ces deux effets peut être illustrée graphiquement. La figure $\mathrm{n}^{\circ} 4$ présente le choix d'heures travaillées d'un bénéficiaire de l'allocation chômage, selon la valeur de son taux de remplacement. Le cadre analytique est celui du modèle micro-économique d'offre de travail. Chacune des courbes correspond à une courbe d'indifférence de l'agent. Dans la situation initiale (taux de remplacement élevé) la contrainte budgétaire est donnée par la droite passant par le point $A$. On a supposé que l'agent ne souhaitait pas exercer d'activité réduite. Dans le cadre du dispositif d'intéressement, une baisse du taux de remplacement abaisse la contrainte budgétaire mais la rend aussi plus pentue : le niveau de ressources en l'absence d'activité est plus faible mais les gains nets retirés de l'activité réduite sont plus élevés à salaire horaire et volume d'heures donnés. On a supposé que, suite à la baisse du taux d'indemnisation, l'in-

Figure 4

Effets d'une variation du taux de remplacement en présence d'intéressement

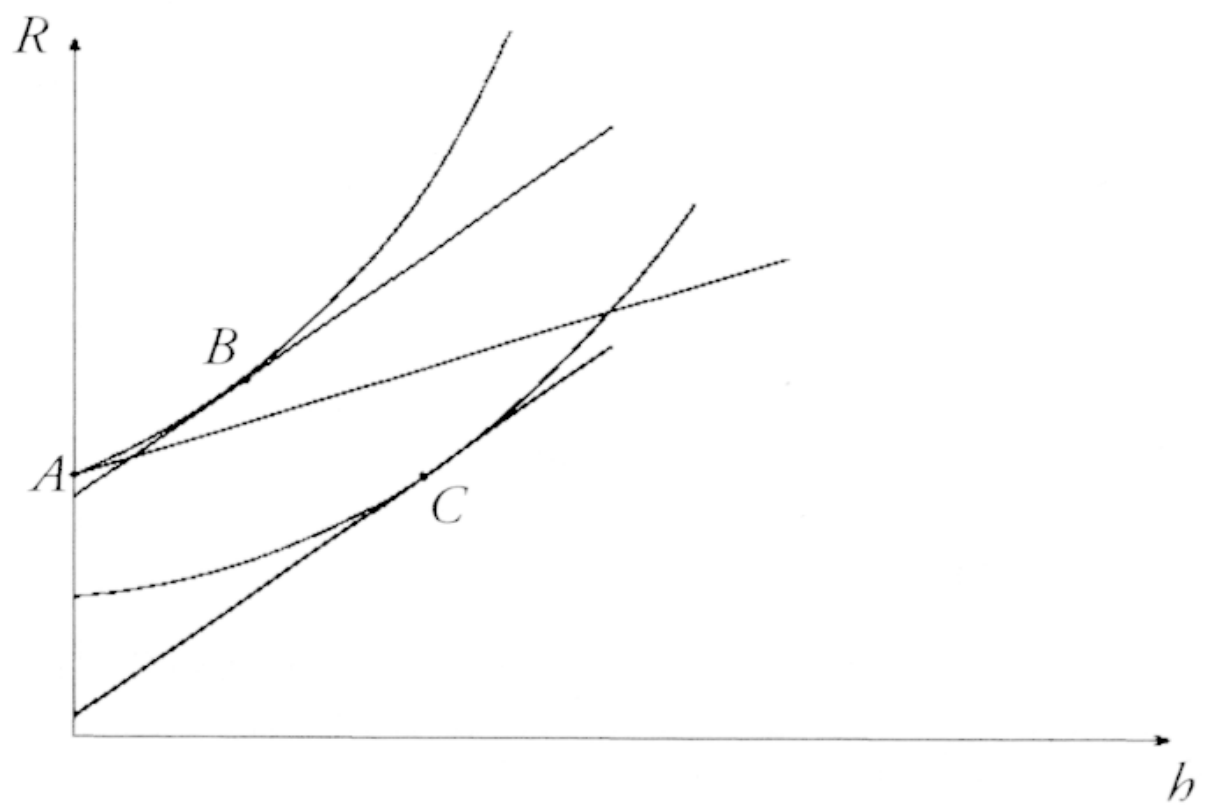


dividu choisirait d'exercer une activité réduite : il choisit le point $C$.

La variation du taux d'indemnisation met alors en jeu deux effets. D'une part, un effet de substitution, correspondant au passage de $A$ à $B$ : comme le revenu tiré de toute heure travaillée en activité réduite augmente, le nombre d'heures que l'individu souhaite travailler augmente, même à niveau de ressources hors activité réduite inchangées. D'autre part, un effet de revenu, correspondant au passage de $B$ à $C$ : comme le revenu hors activité de l'individu diminue, il va compenser cette baisse de revenu par une augmentation de ses heures travaillées même si le salaire horaire effectif (net de l'imposition implicite dans le système de cumul partiel) ne se modifie pas. Face à une baisse du niveau d'indemnisation, cet effet existerait même en l'absence de toute possibilité de cumul.

Il apparaît important de distinguer empiriquement ces deux effets pour mettre à jour l'effet du dispositif d'intéressement. Si l'effet d'une baisse de $q$ transite uniquement par le second effet (effet revenu) alors la possibilité de cumul n'a pas d'incidence sur l'exercice d'une activité réduite et tout l'effet incitatif réside dans la variation du niveau d'indemnisation hors cumul. Dans ce cas, le versement d'une allocation chômage dégressive sans possibilité de cumul aurait les mêmes effets que le dispositif d'intéressement.

$\mathrm{Si}$, au contraire, il existe un effet de substitution important, c'est à dire si la pente de la contrainte budgétaire a une influence forte sur les décisions individuelles, alors il convient de s'interroger sur le taux de cumul qui favorise le plus l'exercice d'activité réduite. De façon évidente, il s'agira du cumul total qui correspond au système de franchise existant dans le cas américain et qui sera discuté ci-dessous.

\section{Mc Call [1996]}

L’article de McCall [1996] apporte quelques éléments complémentaires sur l'effet des possibilités d'intéressement en matière de reprise d'emploi à temps partiel. 
Cette étude examine l'effet des possibilités de cumul au sein du dispositif américain. Dans la plupart des Etats, il est possible de cumuler pleinement les revenus d'un emploi à temps partiel avec les revenus tirés de l'assurance-chômage. L'étendue de ce cumul est limitée par un plafond, le disregard ou franchise. Au-delà du montant de disregard, les revenus supplémentaires sont implicitement taxés à $100 \%$ jusqu'à hauteur de la somme des indemnités et du disregard. Enfin, le fait d'exercer un emploi à temps partiel permet d'allonger la durée pendant laquelle un individu peut prétendre à des indemnités chômage.

Lauteur exploite le fait que le montant du disregard varie d'un Etat à l'autre et que dans certains cas la législation pour un Etat donné a varié au cours de la période étudiée.

En cohérence avec le modèle théorique déjà évoqué, l'auteur estime un modèle économétrique de transition vers l'emploi, dans lequel la transition vers un emploi à temps plein et celle vers un temps partiel sont traitées comme des risques concurrents. ${ }^{32}$

Les résultats indiquent tout d'abord que le système de franchise affecte positivement la sortie du chômage vers un emploi à temps partiel. La probabilité de sortie du chômage vers un emploi de ce type est positivement corrélée au montant du disregard.

Par contre, le montant du disregard est sans effet sur la probabilité de sortie vers des emplois à plein temps. Il convient d'insister sur l'interprétation de ce résultat. Il signifie que, conditionnellement au fait de ne pas trouver un emploi à temps partiel, la probabilité de trouver un emploi à temps plein n'est pas affectée par l'existence du système de franchise. Les effets de substitution d'un effort de recherche d'emploi à temps partiel à un effort de recherche d'emploi à temps plein suggérés dans le modèle théorique ne semblent donc pas jouer. Ces résultats ne signifient pas pour autant que la probabilité de transiter vers un emploi à temps plein, observée dans les données, sera inchangée : elle diminuera dans la mesure où le risque concurrent de retrouver un emploi à temps partiel augmente. 


\section{Effets de l'intéressement sur la sortie du chômage}

\section{Granier et Joutard [1999]}

L'étude de Granier et Joutard analyse l'effet de l'exercice d'une activité réduite sur la probabilité de sortie du chômage. Elle examine conjointement l'effet de la durée de chômage écoulée sur l'exercice d'activité réduite.

Létude considère comme activité réduite tout exercice d'un emploi par un chômeur encore inscrit auprès de l'ANPE. Il s'agit donc d'emplois d'attente occupés pendant la recherche d'un emploi plus satisfaisant, qui ne correspond pas précisément à la définition retenue dans le cadre l'ARAC.

La principale difficulté méthodologique rencontrée tient à un possible " biais d'hétérogénéité " dans l'estimation des interactions croisées entre durée de chômage et exercice d'une activité réduite. L'existence d'une hétérogénéité individuelle non prise en compte biaisera les effets estimés en rendant endogène la durée de chômage, du point de vue de l'exercice d'activité réduite, et l'exercice d'une activité réduite, du point de vue de la probabilité de sortir du chômage.

Plus précisément, supposons qu'il existe une hétérogénéité individuelle intrinsèque tant dans la probabilité de sortir du chômage que dans la probabilité d'exercer une activité réduite. Dès lors que la probabilité de sortie du chômage est positivement (respectivement négativement) corrélée à la probabilité d'exercer une activité réduite :

- En régressant l'exercice d'une activité réduite sur la durée de chômage on sous-estimera (respectivement sur-estimera) l'effet de la durée de chômage sur la probabilité d'exercice d'une activité réduite.

- En régressant la sortie du chômage sur l'exercice d'une activité réduite on sur-estimera (respectivement sous-estimera) l'effet de la durée de chômage sur la probabilité d'exercice d'une activité réduite.

En modélisant conjointement le processus de participation à l'activité réduite et le processus de sortie du chômage et 
en prenant en compte la possibilité de facteurs inobservables, influant simultanément sur chacun de ces deux processus, le modèle estimé permet d'isoler l'effet propre de l'activité réduite sur la sortie du chômage et de la durée de chômage sur l'exercice d'activité réduite.

Le modèle est estimé à partir du fichier historique des demandeurs d'emploi de l'ANPE. La population étudiée est constituée de l'ensemble des demandeurs d'emploi présents dans le fichier, qu'ils soient indemnisés ou non. Pour chaque individu présent dans le fichier, seul est pris en compte le premier épisode de chômage.

L'étude révèle tout d'abord que la durée de chômage influe de façon non monotone sur la probabilité d'exercer une activité réduite un mois donné. Pour les hommes comme pour les femmes, la probabilité d'exercer une activité réduite semble augmenter au cours de la première année de chômage pour atteindre un pic pour une valeur de la durée d'inscription légèrement supérieure à 12 mois. La probabilité moyenne d'exercer une activité réduite un mois donné passerait alors de $6 \%$ à environ $10 \%$. Cette probabilité décroîtrait ensuite pour revenir à un niveau de $6 \%$ après environ 2 mois et demi et tendre vers zéro au-delà. Par ailleurs, la pratique antérieure d'une activité réduite semble influencer positivement la probabilité d'exercer une activité réduite un mois donné ${ }^{34}$.

Pour analyser l'effet de l'exercice d'une activité réduite sur la probabilité de sortie du chômage, les auteurs distinguent effets courants et effets retardés ${ }^{35}$. Les effets estimés apparaissent très variables selon la durée de chômage déjà écoulée. Cependant, des effets positifs de la pratique passée semblent à l'œuvre.

Dans le cas des hommes, les résultats suggèrent que l'effet de la pratique d'activité réduite, dans la période courante, sur le taux de sortie du chômage est très faible (et non significatif) au cours de la première année de chômage. Par contre, il devient significatif et quantitativement important entre le 13ème et le 18 ème mois de chômage, puis à nouveau non significatif. Dans le cas des femmes, l'effet de l'activité réduite courante est positif et significatif seulement entre le $7^{\circ}$ et le $12^{\circ}$ mois de chômage 
et est significativement négatif pour des durées de chômage plus élevées. Enfin, l'effet d'une activité réduite passée sur le taux de sortie du chômage est positif et significatif, dans le cas des hommes comme dans celui des femmes.

Il reste cependant difficile au regard des données de l'article d'avancer une valeur synthétique de l'élasticité de la durée de chômage par rapport à la pratique d'activité réduite.

Les résultats obtenus dans cet article méritent tout d'abord d'être discutés au regard des hypothèses de modélisation retenues. Les variations de l'effet de l'activité réduite selon la maturité de l'épisode de chômage incitent en particulier à une certaine prudence dans la lecture des résultats. Elles doivent être rapprochées des variations propres du taux de sortie du chômage au cours des épisodes de recherche d'emploi. Certaines études ont en effet révélé l'existence de pics discontinus dans le taux de sortie du chômage notamment à l'approche de changements de régimes d'indemnisation ${ }^{36}$.

Il convient alors de se demander dans quelle mesure la spécification retenue pour modéliser l'effet propre de la durée de chômage sur le taux de sortie permet de saisir de façon satisfaisante de tels pics. Si tel n'est pas le cas, il est possible que les variables indicatrices de l'exercice d'une activité réduite pendant la période courante ne saisissent pas l'effet propre de l'activité réduite mais simplement la variation au cours de l'épisode du taux de sortie du chômage. C'est d'autant plus vraisemblable que, d'une part, la montée la plus nette des taux de sortie du chômage semble intervenir au terme d'un an et que, d'autre part, l'exercice d'activité réduite connaît une montée en puissance au cours de la première année de chômage.

\section{Gerfin et Lechner [2002]}

L'étude de Gerfin et Lechner [2002] examine les effets du dispositif d'intéressement à l'œuvre en Suisse. Comme on l'a vu, ce dispositif est très proche de celui en vigueur en France et ne s'en distingue que par l'absence de plafond à la durée mensuelle de travail ouvrant droit au cumul. Les auteurs évaluent l'effet de ce 
dispositif en termes de retour à l'emploi et comparent cet effet à ceux de deux autres mesures de la politique de l'emploi : les programmes de formation et les dispositifs d'emploi public.

L'effet du dispositif, en termes de retour à l'emploi, est mesuré par l'écart entre la probabilité moyenne d'être employé un an après le début de l'emploi en intéressement (ou de la participation à une mesure active de la politique de l'emploi) et la probabilité moyenne d'être employé en l'absence de toute participation à un dispositif de la politique de l'emploi. Compte tenu de l'information très riche sur les caractéristiques individuelles disponible dans les données utilisées ${ }^{37}$, les auteurs appliquent des méthodes d'estimation par matching, valides sous l'hypothèse - vraisemblable dans le cas étudié - d'absence de sélection sur des caractéristiques résiduelles non observables. Ces méthodes permettent de tenir compte d'effets différenciés par sous-population.

Cette étude révèle tout d'abord un effet de sélection positif au sein du dispositif d'intéressement. En moyenne, les individus ayant recours à ce programme semblent avoir un attachement antérieur plus fort au marché du travail. Une fois prise en compte cette hétérogénéité des participants au dispositif d'intéressement, le dispositif semble avoir un effet propre positif et significatif sur la probabilité d'emploi un an après l'entrée. Le taux d'emploi à un an des bénéficiaires de l'intéressement est de six points supérieur à celui des personnes n'ayant participé à aucun dispositif de la politique active. La probabilité d'emploi des bénéficiaires de l'intéressement apparaît aussi fortement et significativement supérieure à celle des participants aux programmes de formation et d'emplois publics. Ces deux derniers programmes ont d'ailleurs un effet négatif sur la probabilité d'emploi des participants, comparativement au fait de ne bénéficier d'aucune mesure active.

Il est enfin possible, à partir des effets estimés au niveau individuel sur les participants effectifs au programme d'intéressement, de prédire l'effet moyen du programme sur la population des participants potentiels. L'exercice révèle que cet effet est au moins égal à celui observé sur la population des participants 
effectifs, signalant ainsi l'absence d'auto-sélection des participants effectifs sur la base des bénéfices attendus.

\section{Ham et Lalonde [1996]}

L'article de Ham et Lalonde [1996] offre quelques enseignements intéressants, à partir de l'étude d'un programme américain d'emploi subventionné. La mesure active de politique de l'emploi évaluée consiste en un programme d'emploi subventionné (National Supported Work demonstation ou NSW) à destination de femmes bénéficiaires des minima sociaux ${ }^{38}$. Les emplois offerts dans ce cadre devaient durer douze mois. Les normes de travail exigées des participantes étaient graduellement augmentées au cours de la période d'emploi. Les salaires offerts dans le cadre de ces emplois étaient en général inférieurs aux salaires du marché.

On note que le taux d'emploi des participantes au programme est, plusieurs mois après la fin du programme, nettement plus élevé (d'environ $25 \%$ ) que celui des personnes de même caractéristiques mais ne participant pas au programme. Il faut remarquer que ce résultat ne provient pas d'un biais de sélection dans le programme dans la mesure où la participation au programme est décidée sur la base d'une affectation aléatoire.

Les auteurs examinent alors par quel biais le programme contribue à un plus fort taux d'emploi des personnes traitées. Ils distinguent deux effets possibles du programme : un effet sur la probabilité de sortir du chômage et un effet sur la probabilité de conserver un emploi une fois sorti du chômage.

Pour répondre à cette question, ils estiment un modèle de transitions entre états sur le marché du travail et modélisent à la fois le risque de sortie de la formation vers l'emploi, le risque de sortie de la formation vers le chômage, le risque de sortie des épisodes ultérieurs de chômage vers l'emploi et le risque de sortie des épisodes d'emploi vers le chômage.

L'estimation du modèle, sans tenir compte des facteurs d'hétérogénéité individuelle inobservables corrélés entre risques, indique que la participation au programme d'emploi subventionné 
diminue le risque de transition vers le chômage une fois un emploi trouvé mais suggère que la participation au programme diminuerait aussi le taux de sortie du chômage vers l'emploi.

Une variante du modèle est alors estimée pour tenir compte de l'hétérogénéité inobservable. Cet enrichissement du modèle affecte de façon importante les effets mesurés du programme. Une fois prise en compte l'hétérogénéité inobservable, l'effet positif de la participation au programme sur les chances de rester dans l'emploi se maintient mais l'effet négatif du programme sur les chances de sortie du chômage disparaît.

Même si cette étude porte sur une expérience différente des dispositifs de cumul allocation-revenu d'activité, ses résultats sont riches d'enseignements. Elle indique tout d'abord qu'une partie des effets à attendre peut dépasser la transition initiale du chômage vers l'emploi : la pratique d'activité réduite peut vraisemblablement affecter le risque de retour au chômage une fois le premier emploi trouvé. Par ailleurs, d'un point de vue méthodologique, cet article suggère aussi que des conclusions robustes sur les effets du programme ne peuvent être obtenues sans un traitement approprié des problèmes d'endogénéité et d'hétérogénéité inobservable.

\section{Effets de l'intéressement sur les conditions d'insertion au marché du travail}

Au-delà de l'effet du dispositif d'intéressement sur les transitions entre les états de chômage et d'emploi, certaines études, essentiellement descriptives, ont analysé les effets de la pratique d'activité réduite sur la nature de l'emploi retrouvé.

\section{Gurgand [1999] et Gurgand et Letablier [1999]}

Gurgand [1999] et Gurgand et Letablier [1999] analysent l'effet de l'exercice d'une activité réduite sur la nature des emplois retrouvés ultérieurement. L'activité réduite est ici définie comme l'exercice d'un emploi avec maintien de l'inscription auprès de 
l'ANPE. La nature des emplois retrouvés est analysée au travers du type de contrat retrouvé (contrat à durée indéterminée, contrat à durée déterminée, intérim ou contrat saisonnier) et du nombre d'heures travaillées. Les auteurs examinent à la fois l'effet global de la pratique d'activité réduite sur ces deux caractéristiques de l'emploi retrouvé et l'effet de la nature de l'emploi exercé en activité réduite (type de contrat) sur les conditions de retour à l'emploi.

La démarche retenue par les auteurs consiste à modéliser la probabilité de transiter vers différents types d'emploi définis par la nature du contrat ou par l'horaire de travail. Les variables observables incluses parmi les déterminants de la probabilité de transition sont l'exercice d'une activité réduite, la nature du contrat en activité réduite, la nature de l'emploi exercé avant le passage par le chômage et un ensemble de caractéristiques socio-démographiques.

Les résultats indiquent que l'exercice d'une activité réduite et la nature de l'activité réduite ont peu d'influence sur les caractéristiques de l'emploi retrouvé. La pratique d'activité réduite ne diminuerait donc pas la probabilité relative de retrouver un emploi en CDI ou un emploi à temps plein et ce quelle que soit la nature de l'activité réduite exercée. Ainsi, il ne semblerait pas exister d'effet stigmatisant de l'activité réduite sur les conditions d'insertion future au marché du travail. L'activité réduite correspondrait donc tout au plus à un déclassement temporaire sans conséquence négative à long terme.

Les conclusions de ces deux études doivent cependant être considérées avec une certaine prudence. Deux aspects des données utilisées en limitent en effet la robustesse et la portée. D'une part, les résultats s'appuient sur un échantillon de taille relativement faible. De ce fait, il est difficile de distinguer, dans les différences de probabilité de transition vers tel ou tel type d'emploi, l'effet spécifique de l'activité réduite de différences résultant d'un simple bruit statistique ou d'erreurs d'échantillonnage. Par ailleurs, comme le souligne Gurgand [1999], les données utilisées souffrent d'une grande imprécision dans l'enregistrement de l'activité réduite. Les données utilisées proviennent d'une 
enquête téléphonique menée à partir d'un échantillon de chômeurs inscrits à l'ANPE. Or, on constate un manque fréquent de coïncidence entre les réponses des individus concernant la pratique d'activité réduite et les enregistrements de l'activité réduite dans les fichiers de l'ANPE. Ceci indique l'existence d'importantes erreurs de mesure dans les données recueillies par l'enquête. En présence de telles erreurs de mesure dans les variables explicatives du modèle, les coefficients estimés dans l'analyse économétriques seront alors systématiquement biaisés dans le sens d'un effet plus faible (en valeur absolue) que l'effet réel et moins significatif.

\section{Canceill et Huyghues Despointes [1999]}

Canceill et Huyghues Despointes [1999] analysent à partir de l'enquête Trajectoire des demandeurs d'emploi les parcours sur le marché du travail consécutifs à l'inscription auprès de l'ANPE. A partir de méthodes statistiques d'analyse des données, ils dégagent parmi la diversité des trajectoires individuelles six principaux types de trajectoires, caractéristiques des différents parcours.

Parmi l'ensemble des parcours d'insertion distingués, ils mettent en évidence l'existence d'un parcours d'accès progressif à l'emploi faisant intervenir une période intérim de recherche d'emploi concomitante à l'exercice d'un emploi. Cette situation se rapproche donc des situations d'activité réduite au sens de l'ANPE, analysées notamment dans Granier et Joutard [1999]. Ce parcours-type est à distinguer des autres trajectoires que sont les trajectoires de chômage de longue durée, d'accès rapide à l'emploi, d'exclusion partielle du marché du travail (marquée par de forts retraits d'activité) et de chômage récurrent.

Dans les parcours d'accès à l'emploi marqués par une période de chômage avec activité réduite, l'activité exercée pendant la période de recherche d'emploi correspond en général à des emplois de courte durée et/ou à temps partiel. Cette période intérim dure en moyenne six mois. Par ailleurs, dans ces parcours d'insertion, il est à noter que le recours à l'activité irrégulière commence dès le premier mois de chômage. 
Ces trajectoires sont par ailleurs caractérisées par une diminution progressive, au cours du temps, de la part des individus connaissant une situation jointe d'emploi occasionnel, de chômage et de recherche d'emploi. D'après les auteurs, la recherche d'emploi semble cesser à mesure que des emplois suffisamment satisfaisants sont trouvés. Les données qui sous-tendent cette conclusion n'apparaissent pas de manière très nette dans l'étude mais il est vraisemblable que l'abandon de la recherche d'emploi est parallèle à une diminution du caractère irrégulier de l'activité. A moyen terme, il apparaît que trois ans après l'inscription auprès de l'ANPE, les deux tiers des individus connaissant ce type de parcours ont retrouvé un emploi qui leur convient, si on en juge par le fait qu'ils ont cessé de rechercher un autre emploi. En outre, les auteurs font remarquer que ce taux de retour à l'emploi à terme est assez proche de celui des individus qui connaissent un retour à l'emploi rapide.

Au regard de cette analyse, il semble donc que l'exercice d'une activité irrégulière correspond à une étape transitoire conduisant, à terme, à une insertion durable dans l'emploi ${ }^{39}$. Les auteurs notent cependant que la nature des emplois retrouvés par les chômeurs exerçant, pendant leur recherche d'emploi, une activité irrégulière reste marquée par une certaine précarité. La part des emplois régis par des contrats temporaires reste en effet importante, même lorsque la période de recherche d'emploi a cessé. Elle est par exemple significativement plus forte que pour les personnes connaissant un parcours d'insertion rapide. De même, le poids des emplois exercés en intérim demeure, à long terme, relativement important, alors que les individus qui suivent ce type d'itinéraire n'ont en général pas eu recours à ce type d'emploi avant leur inscription à l'ANPE.

Compte tenu de la méthode d'analyse utilisée, il reste cependant difficile de tirer ces résultats dans le sens d'un effet causal positif de l'activité réduite en matière d'insertion et de l'absence d'effet négatif en matière de précarisation. Tout au plus les résultats signalent-ils l'existence d'une corrélation entre activité irrégulière intérim et conditions de retour à l'emploi. 


\section{Bilan et perspectives}

Les différentes études consacrées à l'exercice d'une activité réduite révèlent donc les principaux résultats suivants :

- R1 : l'activité réduite est surtout le fait de personnes qui sont entrées en chômage à la fin d'un emploi précaire (mission d'intérim ou contrat à durée déterminée).

- R2 : l'exercice d'une activité réduite prend la plupart du temps la forme d'une activité précaire (mission d'intérim ou contrat à durée déterminée).

- R3 : dans le cas des chômeurs indemnisés, l'exercice d'une activité réduite est influencé par le niveau du taux de remplacement, mais l'effet à l'ouvre (incitations marginales ou niveau de l'indemnisation) n'est pas clairement identifié.

- R4 : l'exercice passé d'une activité réduite semble favoriser la sortie du chômage.

L'exercice d'une activité réduite semble donc relever d'une insertion précaire au marché du travail tant du point de vue de l'origine des bénéficiaires de la mesure d'intéressement que de celui des caractéristiques des emplois occupés en intéressement. Toutefois, au regard des deux derniers résultats, il apparaît que le dispositif d'intéressement ne se limite pas à accompagner le déroulement de telles trajectoires précaires. Certes, le mécanisme d'intéressement a effectivement pour résultat d'encourager la pratique d'activités relativement précaires. Cependant, il semblerait aussi par ce biais favoriser la sortie du chômage. Dans cette optique, un des effets bénéfiques du dispositif d'intéressement pourrait être d'accompagner financièrement le déroulement d'épisodes d'emploi transitoires, eux-mêmes condition d'une insertion plus durable au marché du travail.

Dans l'état des travaux empiriques sur les effets de l'activité réduite, cette lecture des effets du mécanisme d'intéressement reste cependant hypothétique. Un certain nombre de ques- 
tions essentielles à l'étude des effets du mécanisme d'intéressement restent sans réponse dans les études existantes :

- Q1 : Dans quelle mesure le dispositif d'intéressement contribue-t-il à élever le taux d'emploi des bénéficiaires ? Il s'agit là d'une question centrale pour l'évaluation des effets du dispositif d'intéressement. Dans la mesure où ce dispositif vise à améliorer l'insertion au marché du travail des chômeurs indemnisés, on souhaiterait disposer d'une mesure de ses effets à long terme. Il faudrait alors dépasser la simple évaluation des effets sur la probabilité de sortie du chômage. Une première évaluation - en forme réduite - de ces effets pourrait être obtenue en comparant les taux d'emploi à long terme - par exemple deux ou trois ans après l'entrée en chômage - des personnes ayant exercé une activité réduite et de celles n'en ayant pas exercé.

- Q2 : Par quel biais la pratique d'activité réduite favorise-t-elle la sortie du chômage ? Sans évaluer l'incidence de l'activité réduite sur l'accumulation de compétences ou sur les éventuels comportements de discrimination à l'embauche, il est néanmoins possible d'étudier l'effet de la pratique d'une activité réduite sur le comportement de recherche d'emploi ou sur les offres d'emploi reçues par les chômeurs. Les données d'enquête - notamment l'enquête Trajectoire des demandeurs d'emploi de la DARES - fournissent une information très riche sur les comportements de recherche d'emploi qui pourrait être exploitée dans cette optique. On pourrait aussi chercher à déterminer si la participation au dispositif d'intéressement permet une embauche pérenne, sous d'autres formes de contrat, dans les entreprises au sein desquelles l'activité réduite a été exercée.

Par ailleurs, au-delà d'une approche globale des effets de la pratique d'activité réduite, il conviendrait aussi d'examiner de façon plus fine dans quelle mesure les effets de l'activité réduite en matière de sortie du chômage dépendent des caractéristiques de l'emploi exercé. On pourrait ainsi distinguer les effets de l'activité réduite selon qu'elle est exercée dans le cadre d'emplois à durée déterminée, de mission d'intérim ou de contrats emploi- 
solidarité. Là encore, les données d'enquête rendent possible une telle évaluation.

On notera pour finir que le résultat $\mathrm{R} 4$ reste d'une portée limitée dans la mesure où il demeure compatible avec la possibilité que les effets de l'intéressement en matière d'insertion des demandeurs d'emploi transitent par une précarisation ayant à la fois pour effet de diminuer la durée du chômage et d'augmenter le risque de chômage à terme.

- Q3 : La pratique d'une activité réduite a-t-elle une influence sur la nature de l'emploi retrouvé ? Une fois établie l'influence de la pratique d'activité réduite sur la probabilité de sortir du chômage, l'étude des effets sur le type d'emploi retrouvé apparaît fondamentale. D'une part, parce qu'elle permet de distinguer les effets bénéfiques des phénomènes de précarisation. D'autre part, car les effets sur la nature des emplois retrouvés conditionnent à plus long terme le taux d'emploi des bénéficiaires. Si certains des travaux discutés ci-dessus apportent quelques premiers éléments de réponse, ceux-ci mériteraient d'être élargis, confirmés et approfondis par une prise en compte des problèmes d'endogénéité et de sélection.

Cette question pourrait d'abord être abordée d'un point de vue descriptif. Il s'agirait alors d'étudier les caractéristiques des emplois retrouvés à la sortie du chômage en fonction de l'exercice ou non d'une activité réduite. Il serait en particulier intéressant d'examiner l'effet de l'activité réduite sur le type de contrat de travail de l'emploi, sur le salaire perçu et les heures travaillées ainsi que sur la qualification de l'emploi retrouvé. L'ensemble de ces caractéristiques est en principe disponible dans les données d'enquête.

Cet examen des caractéristiques des emplois retrouvés mériterait par ailleurs d'être complété par une étude de l'effet de la pratique d'activité réduite sur la durée des emplois retrouvés après la sortie du chômage. Ceci compléterait les résultats déjà acquis sur les effets en termes de probabilité de sortie du chômage. 
- Q4 : l'effet du dispositif d'intéressement varie-t-il en fonction des caractéristiques du travailleur? Une des limites des résultats obtenus jusque-là est - à l'exception de l'étude de Gerfin et Lechner - de ne pas examiner les effets du dispositif d'intéressement d'une façon différenciée et qui prenne explicitement en compte l'hétérogénéité de la population des bénéficiaires potentiels.

Il est vraisemblable que les effets du dispositif d'intéressement sur l'insertion future varieront en fonction de la qualification et de l'employabilité des bénéficiaires. Tel emploi exercé dans le cadre de l'activité réduite pourra s'avérer " qualifiant" pour des individus à faible employabilité et stigmatisant pour des individus de qualification plus élevée. Cette hétérogénéité des effets d'une même mesure, selon les caractéristiques de la population traitée, a par exemple été mis en évidence par Bonnal, Fougère et Serandon [1997] dans le cas des mesures de formation et d'emploi aidé. Ils ont ainsi montré que ces mesures pouvaient avoir des effets positifs sur l'insertion des individus les moins qualifiés mais avaient au contraire des effets négatifs sur l'insertion des individus plus qualifiés.

Par ailleurs, les travaux sur les effets incitatifs du dispositif mettent l'accent sur la décision d'offre de travail et ignorent tout rationnement quantitatif de l'offre de travail. On peut cependant penser que l'exercice d'une activité réduite résulte non seulement d'une décision d'offre de travail influencée par des incitations financières mais aussi du fait que les individus trouvent des emplois vérifiant les conditions d'intéressement. Pour des individus dont l'employabilité est faible, cette dernière contrainte ne peut être ignorée.

Ces deux remarques plaident alors pour une étude empirique désagrégée dans laquelle les effets d'incitations et d'insertion seraient évalués séparément sur des sous-populations plus homogènes en termes d'employabilité.

- Q5 : le dispositif d'intéressement constitue-t-il une mesure efficace en termes de dépenses publiques ? Cette question élargit le champ d'investigation en posant non seulement le problème 
des effets du dispositif d'intéressement mais aussi celui du coût de la mesure pour les finances publiques. Elle exige d'une part d'avoir mis à jour les effets à long terme de l'intéressement, d'autre part de disposer d'une évaluation des effets de la mesure en termes de dépenses publiques. Or cette évaluation du coût du dispositif d'intéressement risque de s'avérer délicate. Dans le cas de certains dispositifs de la politique de l'emploi, le coût direct peut être relativement simple à définir : il correspondra à l'ensemble des dépenses effectuées dans le cadre de cette mesure. Il n'en est pas de même dans le cas du dispositif d'intéressement qui conduit à moduler le montant des indemnités chômage. Dans ce cadre, le coût direct du dispositif pourrait être donné par la valeur des indemnités versées aux individus en situation de cumul. A contrario, on pourrait aussi considérer que le dispositif instaure un gain pour l'assurance-chômage, dans la mesure où il conduit les individus en activité réduite à ne percevoir qu'une partie de leurs indemnités. On voit alors que même la simple évaluation du coût direct de la mesure est ambiguë et suppose de disposer d'une modélisation des comportements en présence et en l'absence du dispositif.

Pour cette raison, l'examen de l'efficacité du dispositif en termes de dépense publique risque de s'avérer particulièrement complexe. Il serait cependant souhaitable, non seulement de procéder à un tel bilan coût-bénéfices, mais aussi d'établir, sur la base de ce critère, une comparaison du dispositif d'intéressement avec d'autres dispositifs de la politique de l'emploi, en particulier les différents dispositifs d'emplois aidés et de formation.

Au total, il apparaît qu'une analyse des effets du dispositif ARAC passe à la fois par une étude plus complète et à plus long terme de ses effets en matière de transitions sur le marché du travail et par une analyse plus détaillée de son incidence sur les différentes sous-populations bénéficiaires. Les travaux existants mériteraient donc à la fois d'être enrichis par une analyse des- 
104 Nadia Alibay, Arnaud Lefranc

criptive plus fine et par une modélisation plus large de la dynamique d'insertion individuelle. A cet égard, il semble que l'estimation d'un modèle multi-transitions, dans lequel seraient prises en compte à la fois les transitions du chômage vers l'activité réduite, du chômage vers l'emploi et de l'emploi vers le chômage, en distinguant éventuellement différents types d'emploi, permettrait réellement d'aboutir à un diagnostic des effets de cette principale voie d'activation des dépenses d'assurance-chômage.

THEMA, université de Cergy-Pontoise, 33 boulevard du Port, 95011 Cergy, France.

Les auteurs remercient Marc Gurgand et Patrick Pommier pour leurs remarques sur une version préliminaire de ce texte.

Nadia Alibay est doctorante au THEMA.

Adresse : Université de Cergy-Pontoise, 33 boulevard du Port, 95011 Cergy-Pontoise.

Arnaud Lefranc est maître de conférences à l'Université de Cergy-Pontoise et chercheur au THEMA.

Adresse : Université de Cergy-Pontoise, 33 boulevard du Port, 95011 Cergy-Pontoise. 


\section{Notes}

1. Le nombre maximum de bénéficiaires a été d'environ 15000 personnes.

2. On notera au passage que ce questionnement rejoint en partie l'analyse des politiques d'impôts négatifs visant à subventionner l'offre de travail des moins qualifiés. On pourra se reporter à Cahuc [2002] pour une discussion des effets de la prime française pour l'emploi.

3. La condition de durée mensuelle d'activité dans le nouvel emploi a connu plusieurs changements : de 78 heures mensuelles avant 1992, elle a été élevée depuis à 136 heures. Il en est de même du niveau de rémunération autorisant le cumul : $47 \%$ entre 1986 et 1992 ; $80 \%$ entre 1992 et $1994 ; 70 \%$ depuis.

4. Les titulaires d'un contrat emploisolidarité ne sont pas soumis à la limite temporelle du cumul. Ils peuvent bénéficier du cumul jusqu'à épuisement de leur droits aux allocations chômage ou jusqu'à la fin de leur contrat.

5. L'ASS est réservée aux chômeurs ayant épuisé leurs droits aux allocations d'assurance-chômage et l'AI concerne les individus à statut particulier tels que les ex-détenus, les réfugiés politiques, etc.

6. Pour plus de détails, on pourra consulter Unedic [2001a] et Lefresne et Tuchszirer [2001].

7. Dans le cas irlandais, les revenus d'activité ne sont pas déduits du montant de l'indemnité mais les jours travaillés ne sont pas indemnisés.

8. On pourra consulter Laroque et Salanié [1999] pour une analyse plus large des effets désincitatifs du système de transferts sociaux français.

9. Le taux de remplacement net est donné par le ratio des indemnités chômage nettes sur le salaire net dans l'emploi perdu. La différence entre les taux de remplacement nets discutés ici et les taux de remplacement bruts vient de ce que les taux de prélèvements sociaux sur les indemnités chômage diffèrent de ceux appliqués aux revenus salariaux. Pour plus de détails sur la valeur des taux de remplacement nets on se reportera à Brière [1994] et Pommier et Cohen-Solal [2001].

10. Voir sur ce point Szpiro [2000].

11. Ce cas de figure semble empiriquement pertinent comme le révèle l'étude de Bernard et Canceill [2001], à partir d'une enquête par entretien auprès de bénéficiaires de l'ASS.

12. Comme le remarquent Granier et Joutard [1999] et Gurgand [2000], le taux de remplacement dans le système d'intéressement peut être interprété comme un taux d'imposition implicite sur les revenus d'activité en situation de cumul.

13. Pour mémoire, il faut rappeler que le dispositif d'indemnisation de l'Allocation unique dégressive introduisait une décroissance plus graduelle, par paliers de six mois, du taux de remplacement. De ce fait, la croissance des incitations à l'activité réduite avec la durée de chômage apparaissait plus rapidement que dans le cadre de l'ARE.

14. Pour une analyse de l'effet des caractéristiques individuelles sur le taux de sortie du chômage, on pourra notamment se référer à Bonnal et Fougère 
[1990], Joutard et Werquin [1992], Cohen, Lefranc et Saint-Paul [1997] et Joutard et Ruggiero [2000].

15. Pour une étude plus fouillée, on pourra se référer à Forsé [1997] qui étudie les modes d'obtention de l'emploi occupé à partir de l'enquête Emploi 1994.

16. Ces données doivent être interprétées avec une certaine prudence. Il est possible que la rupture du lien professionnel soit, non pas la cause première, mais un symptome additionnel d'une désafiliation professionnelle de l'individu. Dans ce cas, rien ne garantit que le maintien de relations professionnelles permettrait de favoriser le retour à l'emploi.

\section{Voir Mortensen [1986].}

18. Les conséquences théoriques de cette hypothèse pour l'apparition d'un chômage de longue durée sont analysées dans Pissarides [1992] et Coles et Masters [2000].

19. Voir Lefranc [1998] dans le cas de la France et Addison et Portugal [1989] dans celui des Etats-Unis. Ce résultat cohérent avec une baisse du capital humain avec la durée de chômage peut aussi provenir de la décroissance du salaire de réservation.

20. On pourra aussi consulter Magnac [2000] qui établit la substituabilité des mesures de formation et des emplois temporaires, tant en termes de population d'origine que d'insertion à terme dans le cas des travailleurs jeunes.

21. Voir Machin et Manning [1999] pour une présentation plus détaillée.

22. Leur étude ne porte que sur des travailleurs jeunes.

23. Au regard des résultats de l'étude, on pourrait schématiquement opposer deux groupes : une classe d'individus connaissant une insertion durable au marché du travail et une classe d'individus en situation précaire dont la probabilité de sortie du chômage serait plus élevée mais qui connaîtrait une plus forte probabilité de transiter de l'emploi vers le chômage. Dans ce cas, un nombre élevé de passages par le chômage signalerait avant tout au statisticien un facteur sous-jacent : l'appartenance à un segment instable du marché du travail, sans pour autant que les trajectoires passées aient d'effet propre.

24. Les travaux disponibles sur le temps consacré à la recherche d'emploi indiquent, dans le cas anglais, une durée médiane de recherche d'emploi de l'ordre de 5 heures par semaine. Voir Layard, Nickell, Jackman [1991] p. 236.

25. Ce serait par exemple le cas si était mise en place une expérience naturelle avec assignation aléatoire de la possibilité de bénéficier de l'intéressement.

26. Ce point est mis en évidence par Ham et Lalonde [1996].

27. Unedic $[2001 \mathrm{~b}]$ pp. 179 sq.

28. Gurgand [2000], sections 2.1 et 2.2.

29. Du fait de cette corrélation entre durée d'indemnisation et taux de remplacement, l'identification de l'effet spécifique de chacune de ces variables sur la probabilité d'activité réduite est a priori délicate. Gurgand note cependant que la durée de chômage a un effet continu sur la variable d'intérêt alors que le taux d'indemnisation varie de façon discontinue avec la durée de chômage. Cette discontinuité permet l'identification séparée de l'effet propre du taux d'indemnisation.

30. Cet extrait concerne 4265 individus, soit 71301 mois. Néanmoins, seuls 63548 mois ont été traités puisque $11 \%$ des mois correspondaient à des décalages complets. 
31. Ce résultat n'est valable que si la variation des taux de remplacement n'entraîne pas par ailleurs une hausse des mois en décalage complet (et donc une diminution des mois en cumul).

32. Le modèle de transition est estimé à partir des données du Displaced Worker Supplement du Current Population Survey, équivalent américain de l'enquête Emploi de l'Insee. Les données disponibles ne renseignent que le statut au regard de l'emploi, l'existence de droits à des indemnités chômage et l'état de résidence. Cette dernière information permet d'imputer à chaque individu la valeur du disregard potentiel auquel l'individu a droit. On ne sait cependant jamais si l'individu a ou non recours au cumul. Différentes variantes du modèle sont estimées : avec ou sans hétérogénéité inobservable corrélée entre les risques, avec ou sans sélection sur le recours aux indemnités chômage, avec des effets du disregard qui varient selon l'ancienneté de chômage.

33. Le début de l'article peut induire en erreur dans la mesure où les auteurs y discutent le mécanisme de l'ARAC. En revanche, la suite du texte précise que seuls sont exploités, dans les données utilisées, les modules " caractéristique du demandeur », « emploi recherché » et «activité réduite ». Nous en déduisons que l'information sur l'indemnisation du chômage n'est pas prise en compte

34. On notera que les effets estimés de l'ancienneté de chômage et de l'exercice antérieur d'une activité réduite ne sont pas, a priori, explicables par des phénomènes de sélection puisque que le modèle estimé prend en compte l'existence d'hétérogénéité individuelle inobservable.
35. Ils incluent dans leur modélisation du taux de sortie du chômage une indicatrice de l'exercice d'une activité réduite dans la période courante, une indicatrice de l'exercice d'une activité réduite par le passé. Ces indicatrices sont complétées par des variables mesurant l'intensité de l'activité réduite nombre d'heures dans l'exercice courant et nombre de mois passés en activité réduite).

36. Voir par exemple sur ce point le travail de Prieto $[2000 \mid$ pour la France et Meyer [1990] dans le cas des EtatsUnis.

37. Les données incluent l'historique mensuel complet d'emploi, de chômage et de salaires au cours des dix années précédant l'épisode de chômage en cours, les caractéristiques socio-démographiques usuelles, des évaluations subjectives des chômeurs réalisées par les agences publiques de placement et les caractéristiques détaillées de l'emploi recherché.

38. Il s'agit de l'AFDC.

39. Ces résultat méritent d'être rapprochés de ceux de Farber [1990]. Cet auteur examine les conditions de retour à l'emploi des travailleurs licenciés aux Etats-Unis. Il montre que les pertes d'emploi s'accompagnent d'une augmentation significative de la probabilité d'exercer un emploi à temps partiel. La plupart du temps, cet emploi correspond en outre à du temps partiel subi. Cet effet des pertes d'emploi sur la probabilité d'exercer un emploi à temps partiel diminue au cours du temps. Les pertes d'emploi s'accompagnent donc d'un recours transitoire aux activités à temps partiel qui semble s'inscrire dans le cadre d'un parcours de retour à l'emploi. 


\section{Références}

J.T. Addison et P. Portugal [1989] : Job Displacement, Relative Wage Changes and the Duration of Unemployment. Journal of Labor Economics, 7(3) : pp. 281 302.

P. Bel, M. Beraud, G. Canceill, et S. Lemerle [1998] : Les demandeurs d'emploi en activité occasionnelle ou réduite. Premières informations et premières synthèses, DARES, 45.1, novembre.

S. Bernard et G. Canceill [2001] : Le travail occasionnel des bénéficiaires de l'allocation de solidarité spécifique. Premières synthèses, 20.1 .

O. Blanchard et A. Landier [2002]: The Perverse Effects of Partial Labour Market Reform : Fixed-term Contracts in France. Economic Journal, 1 12(480), juin.

L. Bonnal et D. Fougère [1990]: Les déterminants individuels de la durée du chômage. Economie et prévision, 87 : pp. 93-104.

L. Bonnal, D. Fougère et A. Sérandon [1997] : Evaluating the Impact of French Employment Policies on Individual Labour Market Histories. Review of Economic Studies, 64 : pp. 684-713.

A. Booth, M Francesconi et J. Frank [2002] : Temporary Jobs : Stepping Stones or Dead Ends. Economic Journal, 112(480) : F189-F213, juin.

L. Briere [1994]: Calcul des taux de remplacement pour les chômeurs indemnises par l'Unedic. Note Direction de la prévision (94.BD4.58).

Pierre Cahuc [2002] : A quoi sert la prime pour l'emploi? Revue française d'économie, XVI(3) : pp. 3-62.
G. Canceill et H. Huyghes Despointes [1999] : L'inscription à l'ANPE et après : itinéraires de chômeurs. Premières informations et premières synthèses DARES, 37.1, septembre.

D. Cohen, A. Lefranc et G. Saint-Paul [1997] : French Unemployment: A Transatlantic Perspective. Economic Policy, 25 : pp. 265-285, octobre.

M. Coles et A. Masters [2000] : Retraining and Long-Term Unemployment in a Model of Unlearning by Not Doing. European Economic Review, 44(9) : pp. $1801-1822$.

H. Farber [1999] : Alternative and PartTime Employment Arrangements as a Response to Job Loss. Journal of Labor Economics, 17(4).

M. Forsé [1997] : Capital social et emploi. L'Année sociologique, 47(1) : pp. 143181.

D. Fougère et T. Kamionka [1992a] : Mobilité et précarisation sur le marché francais du travail: une analyse longitudinale pour les années 1986-1988. Economie et prévision, 102-103 : pp. 157$177,1992$.

D. Fougère et T. Kamionka [1992b] : Un modèle markovien du marché du travail. Annales d'économie et statistique, 27 : pp. 149-188.

M. Gerfin et M. Lechner [2002] : $A$ Microeconometric Evaluation of the Active Labour Market Policiy in Switzerland. Economic Journal, 1 12(482) : pp. 854893, octobre.

P. Granier et X. Joutard [1999] : L'activité réduite favorise-t-elle la sortie du chômage ? Economie et statistique 321 322 : pp. 133-148. 
M. Granovetter [1974] : Getting a Job : A Study of Contacts and Carreers. Harvard University Press, Cambridge.

M. Gurgand [1999] : Emplois perdus, emplois retrouvés et le passage par l'activité réduite. mimeo, Centre d'études de l'emploi, mars.

M. Gurgand [2000] : Intéressement : problèmes de mesure, problèmes d'incitation. rapport pour la DARES, décembre.

M. Gurgand [2001] : Activité réduite : le dispositif d'incitation de l'Unedic est-il incitatif? doc. de travail $n^{\circ} 12$, Centre d'études de l'emploi, décembre.

M. Gurgand et M.-Th. Letablier [1999] : Travailler et être inscrit au chômage: emploi d'attente ou statut intermédiaire. 4 pages. Centre d'études de l'emploi, (33), mai.

J. Ham et R. Lalonde [1996] : The Effect of Sample Selection and Initial Conditions in Duration Models : Evidence from Experimental Data on Training. Econométrica, 64(1) : pp. 175-205, janvier.

J. Heckman, R. Lalonde et J. Smith [1999]: The Economics and Econometrics of Active Labor Market Programs, in O. Ashenfelter and D. Card, editors, Handbook of Labor Economics, vol. 3A, chap. 31. North-Holland, Amsterdam.

J.J. Heckman et G. J. Borjas [1980] : Does Unemployment Cause Future Unemployment? Definitions, Questions and Answers from a Continuous Time Model of Heterogeneity and State Dependence. Economica, 47(187) : pp. 247-83, août.

$X$. Joutard et M. Ruggiero [2000] : Recherche d'emploi et risques de récurrence du chômage : une analyse des qualifications. Annales d'économie et de statistique. 57 : pp. 239-265.

X. Joutard et P. Werquin [1992] : Les déterminants individuels de la durée du chômage: de l'intérêt de distinguer les emplois stables des emplois précaires. Economie et prévision, 102-103 : pp. 143155.

G. Laroque et B. Salanié [1999] : Breaking Down Female Non-Employment in France. CREST working paper, 9931, juin.

R. Layard, S. Nickell et R. Jackman [1991]: Unemployment : Macroeconomic Performance and the Labour Market. Oxford University Press, Oxford.

A. Lefranc [1998] : What Do You Lose When You Lose Your Job? Theory and Comparative Evidence. mimeo ThemaUniversité Paris $\mathrm{X}$.

F. Lefresne et C. Tuchszirer [2001] : Activités occasionnelles et dynamiques d'insertion des chômeurs : une comparaison de quatre pays européens. Premières informations et premières synthèses, (24.1), juin.

S. Lollivier [2000] : Récurrence du chômage dans l'insertion des jeunes : des trajectoires hétérogènes. Economie et statistique (334) : pp. 49-64.

S. Machin et A. Manning [1999]: The Causes and Consequences of Longterm Unemployment in Europe. In O. Ashenfelter and D. Card, editors, Handbook of Labor Economics, vol. 3C, chap. 47, pp. 3085-3139. North-Holland, Amsterdam.

T. Magnac [2000] : Subsidised Training and Youth Employment : Distinguishing Unobserved Heterogeneity from State Dependance in Labour Market Histories. Economic Journal, 110 (466) : pp. 805837 , octobre.

P. Brian P. McCall [1996] : Unemployment Insurance Rules, Joblessness and PartTime Work. Econométrica, 64(3) : pp. 647-682, mai. 
B. McCormick [1991] : Unemployment Structure and the Unemployment Puzzle. mimeo, The Employment Institute.

B. D. Meyer [1990]: Unemployment Insurance and Unemployment Spells. Econométrica, 58(4) : pp. 757-782, juillet.

D. T Mortensen [1986] : Job Search and Labor Market Analysis, in O. Ashenfelter and R. Layard, editors, Handbook of Labor Economics, vol. 2, chap. 15, pp. 849-919. North-Holland, Amsterdam.

C. Pissarides [1992] : Loss of Skill During Unemployment and the Persistence of Employment Shocks. Quarterly Journal of Economics, pp. 1371-1391.

P. Pommier et M. Cohen-Solal [2001] : L'indemnisation du chômage en 1999 et 2000. Premières Synthèses, 46.1, novembre.

A. Prieto [2000] : L'impact de la dégressivité des allocations chômage sur le taux de reprise d'emploi. Revue économique, 51(3) : pp. 523-534, mai.

D. Szpiro [2000] : L'intéressement à la reprise d'emploi : une imbrication de règles peu transparente. Droit social, juilletaoût.

Unedic [2001] : Dispositifs européens et internationaux d'assurance-chômage. Zoom 2001. Unedic, Paris.

Unedic [2001]: Les allocataires de l'assurance-chômage en activité réduite. Statis, (161): pp. 179-191.

G. van den Berg, A.G. van Lomwel et J van Ours [2001]: Unemployment Dynamics and Age, mimeo Tilburg University, mai.

R. Winter-Ebmer [1991] : Some Micro Evidence on Unemployment Persistence. Oxford Bulletin of Economics and Statistics, 53(1) : pp. 27-43. 